\title{
Preemption Games with Private Information
}

\author{
HUGO A. HOPENHAYN \\ University of California at Los Angeles \\ and \\ FRANCESCO SQUINTANI \\ University of Essex and Universita' degli Studi di Brescia
}

First version received September 2004; final version accepted May 2010 (Eds.)

\begin{abstract}
Preemption games are widely used to model economic problems such as patent races. We introduce private information into these games and allow for this information to stochastically change over time. This reflects, e.g. how R\&D competitors improve their innovations over time and keep these innovations secret before patenting them. The analysis initially appears intractable because of the complexity of the equilibrium updating of beliefs on opponents' information. However, we demonstrate the existence of a class of equilibria and calculate these equilibria in closed form. We find that the expected durations in these equilibria are longer than when players' information is public but, in some cases, shorter than in the collusive outcome. Hence, R\&D secrecy slows down innovation disclosure.
\end{abstract}

Key words: Stopping games, Private information, Patent races

JEL Codes: C73, D82

\section{INTRODUCTION}

The analysis of timing decisions is of prime importance in economic theory. For example, the timing of patent applications determines the rate of disclosure of innovations, and hence welfare assessments of R\&D activities and policies. Such economic problems are typically represented as "preemption games": models where each agent's strategic decision is when to end the game, and there is a first-mover advantage in the pay-offs. One important, but previously neglected, feature of these timing problems is private information: the agents' pay-off-relevant characteristics (which we define as their states) may be only privately known and may stochastically change over time. For example, R\&D competitors improve their innovations over time and keep them secret before filing for a patent. This paper calculates equilibria in preemption games where players' private information states stochastically increase over time. We contrast our results with the analysis of timing games with symmetric information. We find that equilibrium durations are longer when information is private. We then contrast our equilibria to the collusive outcome when players are in a team that aggregates private information and maximizes joint pay-offs. When each player's state influences only her own pay-off, we find that private information equilibrium durations are shorter than in the collusive outcome. In the context of patent 
races, we conclude that secrecy slows down innovation disclosure, possibly to the advantage of $\mathrm{R} \& \mathrm{D}$ competitors.

Incorporating into the analysis of preemption games, the realistic feature that players' private information states change over time generates novel conceptual obstacles. The calculation of equilibrium beliefs, and hence the calculation of the equilibrium, appears intractable. The only available information to a player is that the opponent has not yet ended the game. How should a player update her equilibrium beliefs of the opponent's state and hence of the risk of being preempted? How should higher-order beliefs affect one's choice? ${ }^{1}$ Also, how should equilibrium beliefs evolve over time? If the opponent is still in the game at a late time, should a player believe that the opponent is likely to remain longer or should she believe that she is coming close to end the game?

We provide definitive answers to these questions in a stylized continuous-time framework. In any instant, each player's state is the sum of past state increments that arrive according to identical independent Poisson processes. Conditional on arrival, the value of the state increment is randomly drawn from distributions that are identical and independent across players. Under mild assumptions on the players' pay-offs, we show existence of equilibria where each agent ends the game when her state is above a time-decreasing threshold, and we derive in closed form the ordinary differential equation that determines the equilibrium threshold. The equilibrium beliefs have the following properties. Because the players do not know each other's states, they use calendar time to make inferences on how close the opponent is to ending the game, and hence on the risk of preemption. Over time, each player becomes more afraid that the opponent will soon end the game and becomes more willing to end the game for lower states and pay-offs. To illustrate the substantive meaning of our equilibrium, consider $e$.g. a patent race where an innovation "is in the air": i.e. more than one firm is working on it. As time advances, the competitors become more and more concerned with the risk of preemption and less willing to wait for additional results before applying for a patent.

Having calculated the closed form of the differential equation determining the timedecreasing equilibrium thresholds, we can perform several comparative statics exercises in applications such as patent races. We find the surprising policy prediction that strengthening patent rights may slow down innovation disclosure. Stronger patents increase both the commercial value of current research and the option value of waiting for additional results that improve current innovations. When research is sufficiently costly compared to the development of innovations, it turns out that strengthening patent rights increases the option value for waiting more than the value of current research, thereby inducing firms to procrastinate patent applications.

In order to highlight the implications of private information in preemption games, we compare the time-decreasing threshold equilibrium of our games with the equilibrium of analogous preemption games with public information. Under some regularity assumptions, we find that the expected equilibrium durations are shorter when information is public. When the players' states are common knowledge, the equilibrium unravels. For fear of being preempted, each player becomes willing to end the game at low states. In turn, this makes the opponent even more afraid of preemption and willing to end the game at even lower states. When the opponent's state is private information, the players do not know whether the opponent's state is close to the stopping

1. Note that a player may be willing to delay ending the game only because she believes that there is no risk of preemption. This belief may be induced by the belief that the opponent believes that there is no risk of preemption and hence is willing to delay ending the game. 
threshold or not. This reduces the fear of preemption and stops the equilibrium from unravelling. In the context of patent races, this result implies that secrecy slows down the speed of technological disclosure. When innovation results are more likely to leak out, research firms may become more afraid of preemption and eager to patent innovations of smaller economic significance. $^{2}$

Finally, we study the problem of a team that aggregates players' private information and that maximizes the team's joint pay-off. We find that, as the time spent in the game grows large, competing players become willing to end the game at states for which the team planner would instruct them to continue. Our results are sharper in the case where each agent's state influences only her own pay-off (the private value case). In any time-decreasing threshold equilibrium, the players stop the game too early relative to the collusive outcome. Intuitively, when pay-offs are shared among the players, there is no risk of preemption, and hence game durations are longer than when players compete. In the context of R\&D races, this result implies that, when there are no competition externalities in the innovation market, secrecy is advantageous to $R \& D$ competitors, as it brings duration times closer to the collusive outcome.

This paper is presented as follows. After the literature review, Section 3 presents our general model of preemption games with private information. Time-decreasing threshold equilibria are calculated in Section 4 and compared to equilibria of games with public information in Section 5. The team problem is studied in Section 6. Section 7 relates our results specifically to patent races. Section 8 concludes. Most proofs are in the appendix.

\section{LITERATURE REVIEW}

Preemption games have been studied widely. Among the earliest games ever studied is "Duel" (see e.g. Karlin, 1959). In this simple preemption game, two duellists shoot at each other with accuracy increasing over time. This model has evident military and economic applications, e.g. Binmore (2004) motivates his study as a model of patent races. Possibly, the simplest preemption game is the centipede game, introduced by Rosenthal (1982). In each round, one of two players may either contribute one dollar to the opponent's account or collect the sum accrued in her account and end the game. A referee adds one dollar to the account for each dollar contributed. The equilibrium unravels completely. For fear of being preempted by the opponent, each player closes her account immediately. In contrast to this immediate unravelling, we show that the equilibrium unravels only gradually in our games with private information. ${ }^{3}$

A number of papers model patent races as preemption games. Possibly the earliest ones are by Fudenberg et al. (1983) and Lippman and McCardle (1988). Closer to our work, Weeds (2002) studies a model with symmetric information where the value of an innovation changes

2. As we later discuss, this result may help explain the acceleration in the patenting rate, observed in the data since the mid 1980's (see Kortum and Lerner, 1999; Hall, Jaffe and Trajtenberg, 2001), as well as differences in citation numbers across R\&D industries.

3. Related to preemption games are their mirror-image games, wars of attrition, i.e. timing games with a latemover advantage. Baye, Kovenock and de Vries (1993) apply these games to lobbying. Bulow and Klemperer (1999) generalize the war of attrition to the case where the last $k$ out of $n$ players receive a higher pay-off. Fudenberg and Tirole (1986) model a duopoly war with changing demand as a war of attrition with private information on marginal costs. Décamps and Mariotti (2004) study a war of attrition where the private cost of the irreversible investment in an uncertain project is private information. Krishna and Morgan (1997) study general wars of attrition with private information. Unlike us, none of these papers allows private information to change over time. 
stochastically over time. Similarly, Lambrecht and Perraudin (2003) study a preemption game where two firms own options to buy an asset with value stochastically changing over time, and where strike prices are private information. Unlike our analysis, private information is of private value and constant over time. ${ }^{4}$

Reinganum (1981), Fudenberg and Tirole (1985) and Riordan (1992) study technology adoption and market entry preemption games. Competitors decide the timing of entry in a market or the timing of adoption of a process innovation. Waiting reduces the costs of entry and of innovation adoption but there is a first-mover advantage. Unlike this paper, they do not consider private information. Closer to our work, Abreu and Brunnermeier (2003) study a preemption game modelling the interaction of traders in a financial bubble. Like us, they consider private information of interdependent value: each trader receives some information of when the bubble is likely to burst. Unlike us, they do not allow information to change over time.

By calculating equilibria of timing games with states changing over time, our paper contributes to the literature on learning and experimentation in games. One strand of this literature has studied strategic experimentation in multi-armed bandit models. In Bolton and Harris (1999) and Keller, Cripps and Rady (2005), each player can switch between a safe arm, whose pay-off is known, and a risky arm whose pay-off is unknown. Further, it is assumed that the players' pay-offs are common knowledge. This assumption has recently been lifted by Rosenberg, Solan and Vieille (2007) and Murto and Valimaki (2005). Both papers consider the case where the switch from the risky arm to the safe arm is irreversible. Rosenberg, Solan and Vieille (2007) show that the equilibrium is in cut-off strategies: Each player chooses to switch when her cumulative pay-off from the risky arm is below a threshold, which decreases with the time spent by the opponent experimenting with the risky arm. Murto and Valimaki (2005) show that almost all players keep the risky arm if and only if it yields a higher expected pay-off than the safe arm, but players tend to stay on the risky arm for too long.

Other papers have considered learning and experimentation in models different from multiarmed bandits. Keller and Rady (1999) study optimal experimentation by a monopolist who faces an unknown demand curve subject to random changes. They show that there are two qualitatively different regimes. In the first one, there is extreme experimentation and good tracking of the prevailing demand curve, in the other one, moderate experimentation and poor tracking. Bergemann and Valimaki (1996) consider a buyer who buys a stream of goods of unknown value from sellers who compete in prices over time. They find that all Markov perfect equilibria are efficient, and that prices below marginal cost emerge naturally to sustain experimentation. Bergemann and Valimaki (2000) expand this model to allow for multiple buyers. Assuming that the performance of the new product is publicly observable, they show that the buyers perform excessive experimentation. Keller and Rady (2003) study price setting by duopolists who do not know the perceived degree of product differentiation. They show that price dispersion arises in cyclical fashions, depending on whether the value of information gathered through price dispersion is high or low. Moscarini and Squintani (2004) study a winner-takes-all R\&D race where firms are privately informed about the arrival rate of the invention. Unlike in this paper, equilibrium expenditure in $R \& D$ is smaller than in the collusive outcome, if the team planner is sufficiently impatient.

4. Other papers have highlighted preemption effects in different models of R\&D races. In the "tug-of-war" model of Harris and Vickers (1985), firms take turns in making costly steps towards a "finish line." The equilibrium immediately unravels: once a firm is ahead in the race, its competitors immediately quit. However, this dramatic unravelling disappears when uncertainty is introduced regarding the duration of each step (Harris and Vickers, 1987). Unlike these models, we introduce private information and show that it makes equilibria unravel only gradually over time. 


\section{THE GAME}

Two players, $A$ and $B$ are engaged in the following timing game. The state of each player $i$ at any time $t$ is expressed as $x_{i}(t) \in \mathbb{R}_{+}$, e.g. $x_{i}$ may be the value of player $i$ 's innovation in a R\&D race. Each player's state is private information and evolves according to a compound Poisson process. Specifically, each player has independent Poisson arrivals of state increments of rate $\rho \geq 0$ : at any time $t$, the next arrival $\tau_{i} \geq t$ is distributed according to the cumulative distribution function (c.d.f.) $H\left(\tau_{i} \mid \rho, t\right)=1-e^{-\rho\left(\tau_{i}-t\right)}$. The c.d.f. of the increment $w$-conditional on arrival-is $G$, with support equal to $\mathbb{R}_{+}$. We assume that $G \in \mathcal{C}^{2}$, with bounded density $g$, and that the distribution of increments is log-concave, ${ }^{5}$ i.e.

$$
d \log G(w) / d w=g(w) / G(w) \text { is decreasing in } w .
$$

The process we have just described defines a distribution of the random state $x_{i}$ of each player $i$ at time $t$; we denote the associated c.d.f. by $F\left(t, x_{i}\right)$. Because the support of the c.d.f. $G$ is $\mathbb{R}_{+}$ and $G \in \mathcal{C}^{2}$, the properties of compound Poisson processes make sure that $F\left(t, x_{i}\right) \in \mathcal{C}^{2}$, and we denote the density of $F\left(t, x_{i}\right)$ by $f\left(t, x_{i}\right){ }^{6}$

Each player $i$ incurs a flow cost $c$ to remain in the game. At any point in time $T$, each player $i$ may decide to stop the game and achieve a pay-off $u\left(x_{i}(T)\right)$. This pay-off need not be instantaneous, but it may be obtained in a continuation game separate from the timing game e.g. it may be the present discounted value of the stream of pay-offs associated with a patented innovation. The opponent receives a pay-off $\underline{u}\left(x_{j}(T), x_{i}(T)\right)$. While the first-mover's pay-off depends only on her own state, we allow the second-mover's pay-off to depend on both states. For example, in patent races, both players' pay-offs likely depend on the first-mover patented innovation, which becomes the industry standard. When representing a player's pay-off, we shall denote her own state by $x$ and the opponent state by $y$. By definition of a preemption game, the first mover has an advantage: there is a uniform (possibly small) bound $\varepsilon$ such that $u(x)>\underline{u}(x, y)+\varepsilon$ for any $x, y$. In the event that players stop the game simultaneously at time $T$, each player $i$ receives the pay-off $\bar{u}\left(x_{i}(T), x_{j}(T)\right)=\left[u\left(x_{i}(T)\right)+\underline{u}\left(x_{i}(T), x_{j}(T)\right)\right] / 2$. We assume that both $u$ and $\underline{u}$ are bounded above and twice continuously differentiable. We assume that there are finite positive bounds $M$ and $M_{1}$ such that $0<u^{\prime}<M$, and $0 \leq \underline{u}_{1}<M_{1}$, this weak inequality allows the utility not to depend on one's state when preempted by the opponent. ${ }^{7}$ In

5. For example, increments may be negative exponentially distributed of parameter $\lambda$, i.e. $g(w)=\lambda e^{-\lambda w}$ or gamma distributed with parameters $a$ and $b$, i.e. $g(w)=\frac{1}{b^{a} \Gamma(a)} w^{a-1} e^{-w / b}$.

6. Note that $x_{i}(t)$ can be expressed as $\sum_{k=0}^{K(t)} w_{k}$, where $K(t)$ is Poisson distributed with parameter $\rho t$, and the increments $w_{k}$ are independent identically distributed of distribution $G$. Hence, the density of $x_{i}(t)$ can be expressed as

$$
f\left(t, x_{i}\right)=\sum_{k=1}^{\infty} f^{(k)}\left(x_{i}\right) \frac{(\rho t)^{k} e^{-k \rho t}}{k !},
$$

where $f^{(1)}\left(x_{i}\right)=g\left(x_{i}\right)$, and recursively, $f^{(k)}\left(x_{i}\right)=\int_{0}^{x_{i}} g\left(x_{i}-w\right) f^{(k-1)}(w) d w$ for $n>1$. Further, the time derivative of $F\left(t, x_{i}\right)$ can be derived as

$$
\begin{aligned}
F_{1}(t, x) & =\lim _{\Delta \rightarrow 0} \frac{F(t+\Delta, x)-F(t, x)}{\Delta}=\lim _{\Delta \rightarrow 0} \frac{(1-\rho \Delta) F(t, x)+\rho \Delta \int_{0}^{x} F(t, x-w) G(d w)-F(t, x)}{\Delta} \\
& =\rho \int_{0}^{x}[F(t, x-w)-F(t, x)] G(d w)
\end{aligned}
$$

because in the small time interval $\Delta$, the probability of more than one arrival is infinitesimal. See e.g. Ross (1995).

7. Throughout the paper, the subscripts 1 and 2 refer to derivatives with respect to the first and second argument. 
order to highlight the competitive features of the environment, we also assume that $\underline{u}_{2} \leq 0$ : an increment of the opponent's state cannot increase the second mover's pay-off. ${ }^{8}$ Pay-offs are discounted with rate $r>0$.

For each player $i=A, B$, a history $h_{t}^{i}$ at time $t$ is a weakly increasing path of states $x_{i}(\tau)$ for $0 \leq \tau<t$. As well as her own state path $\left(x_{i}(\tau)\right)_{\tau \leq t}$, the player knows that the opponent did not leave at any time $\tau: 0 \leq \tau<t$. In general, a pure strategy in this game is a historydependent measurable stopping time $\sigma_{i}$, that identifies the earliest moment at which firm $i$ is willing to stop the game given the history $h_{t}^{i}$. Hence, player $i$ ends the game at time $T_{i}\left(h^{i}\right)=$ $\inf \left\{t: \sigma_{i}\left(h_{t}^{i}\right)=t\right\} .{ }^{9}$ To simplify the analysis, we shall focus on symmetric history-independent equilibria: both players adopt the same equilibrium strategy $\sigma_{i}$, function of their own state $x$ and of calendar time $t$ only, and we shall henceforth omit the subscript $i$ whenever there is no ambiguity. Arguments available upon request show that the restriction to history-independent strategy equilibria is without loss of generality.

\section{THE EQUILIBRIUM}

To calculate the equilibrium strategies, we first define the equilibrium value $V(x, t)$, conditional on the opponent not having stopped the game before time $t$, as a function of state $x$ at time $t$. Each player's strategy, $\sigma$, determines a stopping region $S=\{(x, t): \sigma(x, t)=t\}$ and a continuation region $C=\{(x, t): \sigma(x, t)>t\}$. In the stopping region $S$, the equilibrium value $V(x, t)$ corresponds to the value for stopping $u(x)$. In the continuation region $C$, the equilibrium value $V(x, t)$ satisfies the following standard flow equation (see e.g. Dixit and Pindyck, 1994):

$$
r V(x, t)=-c+\lim _{\Delta t \rightarrow 0^{+}} \frac{E[W(x(t+\Delta t), y(t+\Delta t), t+\Delta t) \mid x, t]-V(x, t)}{\Delta t},
$$

where $W(x, y, t)=V(x, t)$ if $\sigma(y(t), t)>t$, i.e. the opponent has not ended the game by time $t$ and $W(x, y, t)=\underline{u}(x, y)$ otherwise.

In equation (1), the expectation is taken both with respect to the increment of the player's own state $x$ and with respect to the opponent's state $y$. At any time $t$, each player conditions her inference with respect to the opponent's state $y(t)$, on the opponent's strategy $\sigma$, and on the information that $T \geq t$ : i.e. that the opponent has not left the game before $t$. This is equivalent to the information that the opponent's state $y(\tau)$ has been in the continuation region $C$ at any time $\tau<t$. While these beliefs are well defined for all $x$ and $t$, in general their calculation is quite cumbersome.

However, we now show that the updating of beliefs is much simpler in equilibria where the frontier between the continuation region $C$ and the stopping region $S$ is a threshold function $z$ continuous and strictly decreasing over time. At any time $t$, each player can base her inference only on the information that the opponent's state $y$ is smaller than $z$ "one instant before $t$ " and can safely ignore all information gathered on the opponent at any previous time $\tau$. We henceforth focus on equilibria described by a continuous and decreasing threshold $z$, that induce equilibrium values $V(x, t)$ differentiable in $t$. We define our equilibrium class of interest as decreasing-threshold regular equilibria.

8. By increasing (decreasing) we mean strictly increasing (decreasing), whenever this is not explicitly qualified.

9. For a general treatment on how to construct stopping time strategies in continuous time games and on their interpretation, see Simon and Stinchcombe (1989). 
Lemma 1. In any decreasing-threshold regular equilibrium, at any time t, the equilibrium belief of either player with respect to the opponent's state $y(t)$ is the c.d.f. $B(y \mid T \geq t)=$ $F(t, y) / F(t, z(t))$.

Proof. Because the frontier between the continuation region $C$ and the stopping region $S$ is a threshold function $z$, the event $T \geq t$ is equivalent to the event that $y(\tau)<z(\tau)$ for all $\tau<t$. Because the equilibrium threshold function $z(\tau)$ strictly decreases over time $\tau$, the inequality $\lim _{\tau \rightarrow t^{-}} y(\tau) \leq \lim _{\tau \rightarrow t^{-}} z(\tau)$ implies that $y(\tau)<z(\tau)$ for all $\tau<t$ for any weakly increasing opponent's innovation state path $y(\tau)$. The opposite implication is obvious. Because the threshold $z$ is continuous, $\lim _{\tau \rightarrow t^{-}} z(\tau)=z(t)$. Because the probability that a state increment occurs exactly at time $t$ is zero, $\operatorname{Pr}\left(\lim _{\tau \rightarrow t^{-}} y(\tau) \leq z(t)\right)=\operatorname{Pr}(y(t) \leq z(t))$. The result then follows from the definition of conditional probability. \|

Armed with this crucial lemma, we can now proceed to simplify equation (1), specialized to a decreasing-threshold regular equilibrium. ${ }^{10}$ The rate of expected change on the right-hand side has four components. First, there is the effect of time advancing without an increase in one's own state and without the opponent ending the game, $V_{2}(x, t)$. Second, with probability $\rho \Delta t$, the player's own state $x$ can increase by some random increment $w$ : the corresponding expected increase in value per unit of time is thus $\rho \int_{0}^{\infty}[V(x+w, t)-V(x, t)] G(d w)$. Third, the opponent may end the game without experiencing an increase in her state because $z(t)$ crosses the opponent's current state from above, causing the player under consideration a change in value from $V(x, t)$ to $\underline{u}(x, z(t))$. The conditional probability that this happens by time $t+\Delta t$ is $[F(t, z(t))-F(t, z(t+\Delta t)] / F(t, z(t))$. Dividing by $\Delta t$ and taking the limit, we obtain a third term equal to $-[\underline{u}(x, z(t))-V(x, t)] f(t, z(t)) z^{\prime}(t) / F(t, z(t))$. Finally, the opponent may end the game because a state increment takes her state above the threshold. The probability of any such an increment is again $\rho \Delta t$, and given the opponent's current state $y$, the increment must be at least of size $z(t)-y$. Averaging over all such increments $w$ and the unknown state $y$ of the opponent, we obtain a final term equal to $\left.\rho \int_{0}^{z(t)} \int_{z(t)-y}^{\infty}[\underline{u}(x, y+w))-V(x, t)\right] G(d w) F(t, d y) /$ $F(t, z(t))$.

Adding all these terms up, we see that equation (1) is equivalent to

$$
\begin{aligned}
& r V(x, t)=-c+V_{2}(x, t)+\rho \int_{0}^{\infty}[V(x+w, t)-V(x, t)] G(d w) \\
& \left.-[\underline{u}(x, z(t))-V(x, t)] \frac{f(t, z(t))}{F(t, z(t))} z^{\prime}(t)+\rho \int_{0}^{z(t)} \int_{z(t)-y}^{\infty}[u(x, y+w))-V(x, t)\right] G(d w) \frac{F(t, d y)}{F(t, z(t))} .
\end{aligned}
$$

In any symmetric decreasing-threshold regular equilibrium, the best response to an opponent playing according to a time-decreasing threshold $z$ is to play according to $z$. This feature allows us to evaluate the flow equilibrium equation (2) at $x=z(t)$, for any $t$. We impose the value matching and smooth pasting conditions,

$$
V(z(t), t)=u(z(t)), \quad V_{2}(z(t), t)=\frac{\partial}{\partial t} u(z(t))=0 .
$$

We rearrange equation (2) and obtain that any decreasing equilibrium threshold $z$ solves the following differential equation:

$$
[u(z(t))-\underline{u}(z(t), z(t))] \frac{f(t, z(t))}{F(t, z(t))} z^{\prime}(t)=\phi(t, z(t)),
$$

10. In the interest of brevity, here we present only an intuitive sketch of the derivations. A formal proof is available upon request. 
where for any $(t, z)$,

$$
\begin{aligned}
\phi(t, z) \equiv & r u(z)+c-\rho \int_{0}^{\infty}[u(z+w)-u(z)] G(d w) \\
& -\rho \int_{0}^{z} \int_{z-y}^{\infty}[\underline{u}(z, y+w)-u(z)] G(d w) \frac{F(t, d y)}{F(t, z)} .
\end{aligned}
$$

We shall now introduce regularity conditions to demonstrate existence of decreasing-threshold regular equilibria and provide a partial characterization result. The first set of conditions is as follows.

Condition 1. For any $x, y, u^{\prime}(x) \geq \underline{u}_{1}(x, y)$.

Condition 2. For any $x, u^{\prime \prime}(x) \leq 0$.

In words, Condition 1 requires that each player's pay-off dependence in her own state is larger when she is the first mover. Condition 2 imposes weak concavity on the first-mover's pay-off. Conditions 1 and 2 allow us to establish that the best response of each player to any opponent's strategy is a strategy that can be represented as a time-dependent threshold function $z$. Specifically, for any time $t$, there is a unique threshold $z(t)$ such that continuing is optimal if $x<z(t)$ and stopping is optimal if $x>z(t)$. Given this result, a decreasing-threshold regular equilibrium exists as long as there is a strictly decreasing and well-defined solution $z$ to the differential equation (4), which induces a value $V(x, t)$ differentiable in $t$. This result will be established with the aid of the two following conditions.

Condition 3. $(r+2 \rho) u(0)<-c+\rho \int_{0}^{\infty}[u(w)+\underline{u}(0, w)] G(d w)$.

Condition 4. $\lim _{x \rightarrow \infty}(r+\rho) u(x)>-c+\lim _{x \rightarrow \infty} \rho \int_{0}^{\infty} u(x+w) G(d w)$.

Conditions 3 and 4 , while apparently complex, are in fact fairly innocuous boundary conditions. Condition 3 only makes sure that the players are willing to enter the game for low values of $x$, it can be understood as a normalization condition on $u$ and $\underline{u}$. Condition 4 stops players from remaining in the game forever for high values of $x$. Given that $u^{\prime \prime} \leq 0$, this is a mild restriction.

The final set of conditions is as follows.

Condition 5. For any $x, y, \underline{u}_{22}(x, y) \leq 0$.

Condition 6. For any $x, y, \underline{u}_{12}(x, y) \leq 0$.

Conditions 5 and 6 have a simple interpretation: they require that the second-mover's payoff is concave in the opponent's state and satisfies a single-crossing condition. These conditions allow us to establish the properties of all decreasing-threshold regular equilibria for early and late stages in the game. We will show that for $t \rightarrow 0$, the threshold function $z$ converges to $\bar{z}$, the smallest $z$ that solves:

$$
r u(z)=-c+\rho \int_{0}^{\infty}[u(z+w)-u(z)] G(d w)+\rho \int_{z}^{\infty}[\underline{u}(z, w)-u(z)] G(d w) .
$$

For $t \rightarrow \infty$, the threshold function $z$ converges to $\underline{z}$, the smallest $z$ that solves:

$$
r u(z)=-c+\rho \int_{0}^{\infty}[u(z+w)+\underline{u}(z, z+w)-2 u(z)] G(d w) .
$$

The existence and characterization results we have so far described are formally presented in the following theorem. ${ }^{11}$

11. We thank the referees, and especially the Editor, Bernard Salanie', for helping us completing the proof of this result. 
Theorem 1. Under Conditions $1-4$, there exists a decreasing-threshold regular equilibrium. Adding Conditions 5 and 6, in any decreasing-threshold regular equilibrium, the threshold function $z(t)$ converges to $\bar{z}$ as time $t$ converges to zero and $z(t)$ converges to $\underline{z}$ as $t$ converges to infinity.

The main steps in the proof of existence of decreasing-threshold regular equilibria are as follows. After concluding that the best response of each player to any opponent's strategy is a strategy that can be represented as a time-dependent threshold function $z$, we turn to establish that there is a strictly decreasing and well-defined solution $z$ to the differential equation (4). To prove this result, for any $t$, we let $\hat{z}(t)$ be the smallest solution of equation $\phi(t, z)=0$. We note that the flow benefit to remaining in the game decreases as the opponent's state $y$ increases. As the expectation of the opponent's state $y$ increases in time $t$, the player's incentive to stop the game becomes stronger as time goes by. As a result $\hat{z}(t)$ is strictly decreasing in $t .{ }^{12}$ For every $T$, we consider the solution $z$ of the differential equation (4) that coincides with $\hat{z}(T)$ at $T$. We show that this solution is strictly decreasing for all $t<T$. By taking $T$ to infinity, we have constructed a solution $z$ that is strictly decreasing for all $t$. The existence of a decreasingthreshold regular equilibrium is then concluded by verifying that the solution $z$ induces a value $V(x, t)$ differentiable in $t$.

Further, we show that under Conditions 1-6, equation (6) has a unique solution. It follows that any strictly decreasing solution $z(t)$ to the differential equation (4) must satisfy $\lim _{t \rightarrow \infty} z(t)=z$. Since any admissible solution $z$ to equation (4) can never cross the function $\hat{z}$, or else it would be strictly increasing over a non-degenerate interval, this also implies that any continuous strictly decreasing solution $z(t)$ to the differential equation (4) must satisfy $\lim _{t \rightarrow 0} z(t)=\bar{z}$.

The initial threshold $\bar{z}$ equalizes the option value for waiting and the incentive to preempt, when the opponent has just entered the game and has not yet received any state increment. As the chance that the opponent's state $y$ will soon cross the stopping threshold $z$ is small, the player is not particularly afraid of preemption. This impedes the unravelling process and makes the initial threshold relatively large. The asymptotic threshold, $\underline{z}$, equalizes the option value of waiting with the incentive to preempt, supposing that the opponent will surely stop the game if receiving one single state increment, of any value. As time goes by, the chance that the opponent is about to stop the game, although she has not yet done so, becomes larger and larger. As the risk of preemption reaches its maximum, the player's willingness to anticipate stopping is largest, and the unravelling process makes the asymptotic threshold relatively small.

In the Section 5, we contrast the equilibrium unravelling over time of preemption games involving private information, with the immediate equilibrium unravelling of games involving public information.

\section{PUBLIC INFORMATION}

This section compares the equilibria of our games involving private information, with equilibria of analogous games involving public information. We study the framework introduced in Section 3 , with the only modification being that at each time $t$, the histories $h_{t}^{A}$ and $h_{t}^{B}$ are common knowledge. Again, we focus on equilibria where the strategies $\sigma_{i}$ depend only on the current

12. Under some additional conditions, the results of the paper extend to the case where the first-mover's pay-off $u$ depends on the opponent's state, provided that the function $\hat{z}(t)$ defined in the extended model is strictly decreasing. The interested reader may request a full derivation from the authors. 
states $(x, y)$ and not on the entire histories of increments $\left(h_{t}^{i}, h_{t}^{j}\right)$. Omitted derivations, available upon request, show that this assumption is without loss of generality.

The first, immediate, observation is that, because $u(x)>\bar{u}(x, y)>\underline{u}(x, y)$ for any $(x, y)$, if the opponent stops the game at any state $\left(x^{A}, x^{B}\right)$, then the unique best response at the same state $\left(x^{A}, x^{B}\right)$ is to stop the game. This observation yields two important results. The first one is that the players always simultaneously stop the game in equilibrium. ${ }^{13}$ The second one is that there are multiple equilibria in the public information game. For every state $\left(x^{A}, x^{B}\right)$, even if each player would prefer to stay in the game if the opponent did not stop at $\left(x^{A}, x^{B}\right)$, there is an equilibrium where both players coordinate on stopping at $\left(x^{A}, x^{B}\right)$. For any strategy profile $\sigma$, we describe the set $C(\sigma)$ of states $\left(x_{A}, x_{B}\right)$ such that the strategies $\sigma$ prescribe that the players do not stop the game: $\sigma_{A}\left(x_{A}, x_{B}, t\right)>t$ and $\sigma_{B}\left(x_{B}, x_{A}, t\right)>t$, for every $t$. We wish to compare the equilibria of a private information preemption game with the equilibria $\sigma$ of the analogous public information game with the largest set of states $C(\sigma)$.

A profile $\sigma$ is an equilibrium if and only if for each player $i$, a best response to the opponent's strategy $\sigma_{j}$ is to wait at any state $\left(x^{A}, x^{B}\right)$ such that $\sigma$ prescribes not to end the game. Formally, fix a strategy profile $\sigma$. Consider any player $i$ and state $(x, y)$, and let the "best-response" value at $(x, y)$ be $V^{i}(x, y)$ equal to $u(x)$ in the region where the best response to $\sigma$ is to stop the game, thus satisfying the following flow equation in the waiting region:

$$
\begin{aligned}
r V^{i}(x, y)= & -c+\rho \int_{0}^{\infty}\left[V^{i}(x+w, y)-V^{i}(x, y)\right] G(d w) \\
& +\rho \int_{0}^{\infty}\left[V^{i}(x, y+w)-V^{i}(x, y)\right] G(d w) .
\end{aligned}
$$

It is easy to verify that this is a standard Bellman equation for a discounted dynamic program (see e.g. Dixit and Pindyck, 1994). Hence, $\sigma$ is an equilibrium if and only if, for each player $i$, $V^{i}\left(x^{i}, x^{j}\right)>u\left(x^{i}, x^{j}\right)$ for every state $\left(x^{A}, x^{B}\right)$ such that $\sigma_{i}\left(x^{i}, x^{j}, t\right)>t$.

We now introduce the regularity conditions that will deliver our main result that expected durations are longer when information is private.

Condition 7. For any $(x, y), u^{\prime}(x)-\underline{u}_{1}(x, y) \geq-\underline{u}_{2}(x, y)$.

Condition 8. For any $(x, y), u^{\prime}(x)-\underline{u}_{1}(x, y) \geq-\underline{u}_{2}(y, x)$.

Condition 7 requires that the change in each player's first-mover advantage $u(x)-\underline{u}(x, y)$ with respect to her own state, $x$, is larger than the change in the first-mover advantage with respect to her opponent's state, for any pair of states $(x, y)$. Condition 8 requires that the change of the first-mover advantage $u(x)-\underline{u}(x, y)$ with respect to the player's own state when the states are $(x, y)$ is larger than the change of $u(y)-\underline{u}(y, x)$ with respect to the opponent's state when the states are $(y, x)$, i.e. when the states are interchanged between the players.

The next result shows that, under Conditions $1-8$, the set $C(\sigma)$ is bounded above by the threshold function $x_{A}+x_{B}=2 \underline{z}$ in any equilibrium $\sigma$. As the processes of states $x_{A}(t)$ and $x_{B}(t)$ are independent, the expected time for the states $\left(x_{A}(t), x_{B}(t)\right)$ to exit the set $C(\sigma)$ is smaller than the expected time for either process $x_{i}(t)$ to reach $\underline{z}$. This implies that the expected duration of the public information game is smaller than the expected time needed for either, or both, of the players to reach the asymptotic threshold $\underline{z}$. In this sense, we conclude that the

13. In fact, there are two possible scenarios for this game: either the player that had the last arrival has a time advantage and stops the game first (which would correspond to the limit as the information disclosure lag goes to zero) or both players stop simultaneously, by which we mean that each stops first with equal probability. The theorem derived in this section applies to both scenarios, but for simplicity in our presentation, we only consider the latter case. 
equilibrium of the public information game immediately unravels to the threshold that is reached only asymptotically in the equilibrium of the private information game.

Theorem 2. Suppose that the histories $\left(h_{t}^{A}, h_{t}^{B}\right)$ are public at any time $t$ Under Conditions $1-8$, for any equilibrium $\sigma$, the set $C(\sigma)$ is contained in $\left\{\left(x_{A}, x_{B}\right): x_{A}(t)+x_{B}(t) \leq 2 \underline{z}\right\}$. Hence, $E\left[\inf \left\{\tau:\left(x_{A}(\tau), x_{B}(\tau)\right) \notin C(\sigma)\right\}\right]<E\left[\inf \left\{\tau: x_{A}(\tau) \geq \underline{z}\right.\right.$ or $\left.\left.x_{B}(\tau) \geq \underline{z}\right\}\right]$.

This concludes the comparison of preemption games with private and public information. We have found that equilibrium durations are longer under private information. In Section 6, we compare private information equilibrium durations with collusive durations.

\section{THE TEAM'S PROBLEM}

This section compares the equilibrium strategies of our private information games with the collusive policies that maximize the sum of the players' pay-offs under perfect information. As an illustration, the players may be joined in a team that aggregates private information, maximizes the joint pay-off and divides it equally.

For clarity of exposition, we assume that it is always optimal for the player with the largest state to end the game: $u\left(x_{A}\right)+\underline{u}\left(x_{A}, x_{B}\right)>u\left(x_{B}\right)+\underline{u}\left(x_{B}, x_{A}\right)$ if and only if $x_{A}>x_{B}$. Hence, we let the team's pay-off be

$$
u^{*}\left(x_{A}, x_{B}\right)= \begin{cases}u\left(x_{A}\right)+\underline{u}\left(x_{B}, x_{A}\right) & \text { if } x_{A}>x_{B}, \\ u\left(x_{B}\right)+\underline{u}\left(x_{A}, x_{B}\right) & \text { if } x_{B}>x_{A}\end{cases}
$$

because the team planner optimally chooses the first mover to maximize the joint pay-off. To simplify the exposition, we assume that $u^{*}$ is twice continuously differentiable: this requires that the left and right derivatives are the same at $x_{A}=x_{B}$.

We focus on policies $\sigma$ where the decision to stop the game depends only on the states $\left(x_{A}, x_{B}\right)$ and not on the whole history of state increments; omitted arguments show that this restriction is without loss of generality. We denote as $V\left(x_{A}, x_{B}\right)$ the team's value in the game for a pair of states $\left(x_{A}, x_{B}\right)$. In the region where the planner makes the players stop, $V\left(x_{A}, x_{B}\right)=$ $u^{*}\left(x_{A}, x_{B}\right)$. In the continuation region, $V\left(x_{A}, x_{B}\right)$ satisfies the flow equation:

$$
\begin{aligned}
r V\left(x_{A}, x_{B}\right)= & -2 c+\rho\left[\int_{0}^{\infty} V\left(x_{A}+w, x_{B}\right) G(d w)-V\left(x_{A}, x_{B}\right)\right] \\
& +\rho\left[\int_{0}^{\infty} V\left(x_{A}, x_{B}+w\right) G(d w)-V\left(x_{A}, x_{B}\right)\right] .
\end{aligned}
$$

We study the collusive outcome under the following conditions (for consistency with the previous conditions, we express them in terms of the primitive pay-offs $u$ and $\underline{u}$ ).

Condition 9. For any $(x, y), u^{\prime}(x)+\underline{u}_{2}(y, x)>0$.

Condition 10. For any $(x, y), \underline{u}_{11}(x, y) \leq 0$.

Condition 9 requires that the aggregate pay-off $u^{*}\left(x_{A}, x_{B}\right)$ is increasing in both players' states $x_{A}$ and $x_{B}$, i.e. $u_{1}^{*}\left(x_{A}, x_{B}\right)>0$ and $u_{2}^{*}\left(x_{A}, x_{B}\right)>0$. Condition 10 , concavity of the secondmover's pay-off in her own state, implies together with $u^{\prime \prime} \leq 0$ (Condition 2) and $\underline{u}_{22} \leq 0$ (Condition 5), that the aggregate pay-off $u^{*}\left(x_{A}, x_{B}\right)$ is concave in both arguments $x_{A}$ and $x_{B}$, i.e. $u_{11}^{*}\left(x_{A}, x_{B}\right) \leq 0$ and $u_{22}^{*}\left(x_{A}, x_{B}\right) \leq 0$. Note also that $u^{*}$ inherits the single-crossing condition $u_{12}^{*}\left(x_{A}, x_{B}\right) \leq 0$ from Condition 6 . 
Under these conditions, we now show that the team's stopping time is characterized by a stopping boundary $z^{*}$. Whenever the states $\left(x_{A}, x_{B}\right)$ are above this boundary, the social planner instructs the player with the largest state to stop the game. Due to the symmetry of the problem, the boundary is symmetric with respect to the 45 degree line.

Lemma 2. Under Conditions 2, 5, 6, 9, and 10, there is a unique stopping boundary $z^{*}: x_{B} \mapsto$ $x_{A}$, such that continuing is optimal if and only if $x_{A}<z^{*}\left(x_{B}\right)$, and $V\left(z^{*}\left(x_{B}\right), x_{B}\right)=u^{*}\left(z^{*}\left(x_{B}\right)\right.$, $\left.x_{B}\right)$. The stopping boundary $z^{*}$ is strictly decreasing in $x_{B}$ and symmetric, i.e. $x_{A}=z^{*}\left(x_{B}\right)$ if and only if $x_{A}=. z^{*} \cdot{ }^{-1}\left(x_{B}\right)$.

The proof of the first part of this lemma is similar to the proof that, in games with private information, each player's best response in any regular decreasing-threshold equilibrium can be represented by a threshold function (Lemma A.1 in the appendix). The conditions $u_{1}^{*}\left(x_{A}\right.$, $\left.x_{B}\right)>0$ and $u_{2}^{*}\left(x_{A}, x_{B}\right)>0$ make sure that the boundary $z^{*}$ is strictly decreasing. This is the key feature of $z^{*}$ that allows us to calculate it. For state $x_{B}$, we impose that at the boundary $z^{*}\left(x_{B}\right)$, the optimal value $V\left(z^{*}\left(x_{B}\right), x_{B}\right)$ equals $u^{*}\left(z^{*}\left(x_{B}\right), x_{B}\right)$, the value for stopping the player with the largest state. The optimal value $V\left(z^{*}\left(x_{B}\right), x_{B}\right)$ can be explicitly calculated in the waiting region because the boundary $z^{*}$ is strictly decreasing, and hence if either player $A$ or $B$ receives a further state increment $w$, the associated optimal values are $V\left(z^{*}\left(x_{B}\right)+w, x_{B}\right)=u^{*}\left(z^{*}\left(x_{B}\right)+\right.$ $\left.w, x_{B}\right)$ and $V\left(z^{*}\left(x_{B}\right), x_{B}+w\right)=u^{*}\left(z^{*}\left(x_{B}\right), x_{B}+w\right)$, respectively. ${ }^{14}$ With these substitutions, we obtain that, for any state $x_{B}$, the boundary $z^{*}\left(x_{B}\right)$ solves:

$$
\begin{aligned}
(r+2 \rho) u^{*}\left(z^{*}\left(x_{B}\right), x_{B}\right)=-2 c & +\rho \int_{0}^{\infty} u^{*}\left(z^{*}\left(x_{B}\right)+w, x_{B}\right) G(d w) \\
& +\rho \int_{0}^{\infty} u^{*}\left(z^{*}\left(x_{B}\right), x_{B}+w\right) G(d w) .
\end{aligned}
$$

We conclude the welfare analysis by comparing the optimal stopping boundary $z^{*}$ with the equilibrium thresholds $\bar{z}$ and $\underline{z}$. We show that in any regular decreasing-threshold equilibrium at late stages of the game, the players exit at states that are too low relative to the collusive outcome. This is also true at early stages in the game, and hence in the whole game, in the pure private value case (where $\left.\underline{u}_{2}=0\right) .{ }^{15}$ We let $Q$ be the set of states $\left(x_{A}, x_{B}\right)$ such that the planner does not stop the game, i.e. $x_{A}<z^{*}\left(x_{B}\right)$.

Theorem 3. Under Conditions 1-6, 9, and 10, the set $Q$ of states for which the planner does not stop the game is a superset of $[0, \underline{z}] \times[0, \underline{z}]$, the set of states for which neither player stops the game in a decreasing-threshold regular equilibrium for $t \rightarrow \infty$. When $\underline{u}_{2}=0$, the set $Q$ is also a superset of $[0, z(t)] \times[0, z(t)]$, for all $t$.

The intuition behind this result stems from the observation that the social planner is not afraid of preemption. At any moment in time, for any state $\left(x_{A}, x_{B}\right)$, the planner may decide which player should stop the game to maximize the joint pay-off. Since the team planner is not concerned that the players may try to preempt each other, she optimally sets a generous stopping policy. In any regular decreasing-threshold equilibrium, the players attempt to preempt each other. Neither of them internalizes the negative externality that her preemption imposes on

14. If instead of instructing the player with the highest state to stop the game, the team planner continued the game after any state increment $w$, it would not be feasible to calculate $z^{*}$

15. Beyond the private value case, it can be shown that the comparison between the optimal threshold $z^{*}$ and the equilibrium upper bound $\bar{z}$ is indeterminate. 
the opponent. Instead, as they fear each other's preemption, they wind up anticipating exit to a greater and greater extent. This results in exit times smaller than in the collusive outcome.

This result concludes our general analysis of preemption games with private information changing over time. Our general analysis is of wide applicability. In Section 7, we present an application to patent races.

\section{AN APPLICATION: PATENT RACES}

Suppose that the two players are firms who conduct research activity at a flow cost $c$. The amount of resources initially devoted to research activity is common knowledge and determines the innovation hazard rate, $\rho$, and the innovation distribution function, $G .{ }^{16}$ Over time, the research activity improves the value of a patentable innovation. The state $x_{i}(t)$ of firm $i$ corresponds to the value of $i$ 's innovation at time $t$. Each firms' innovation improvements are kept secret until patented. At any time $T$, each firm $i$ may end the patent race by patenting and developing the innovation $x_{i}(T)$. The patenting firm receives the pay-off $u\left(x_{i}(T)\right)$ and the competitor receives the pay-off $\underline{u}\left(x_{j}(T), x_{i}(T)\right) .{ }^{17,18}$ We consider the functional form specification $u(x)=$ $\alpha v(x)-c_{0}$. We single out a cost $c_{0}$ for developing the innovation, and we introduce the parameter $\alpha$ that measures the fraction of innovation value appropriated by the innovator. We assume that the regularity Conditions 1-10 are satisfied, and that $\alpha v(0)>c_{0}$. When one is ahead in the race, the option of dropping out is dominated by the option of patenting one's own innovation. This assumption allows us to focus the analysis on the patenting choice.

Our equilibrium characterization hinges on Theorem 1 . The equilibrium threshold function $z$ is decreasing in time. As the patent race extends further in time, each firm becomes more fearful of being preempted and willing to patent less valuable innovations. Having calculated the differential equation determining the threshold function $z$, we can study the relationship between the equilibrium strategies and a number of variables of interest. We find that the equilibrium threshold function $z$ is increasing in the cost of developing the innovation $c_{0}$ and decreasing in the flow cost of research $c .{ }^{19}$ These results are intuitive: if the cost of staying in the race increases, each firm is willing to stop earlier, whereas if the cost of stopping and developing the innovation increases, each firm chooses to wait longer.

Quite unexpectedly, making patent rights stronger does not necessarily make innovation disclosure faster. Increasing $\alpha$, the fraction of innovation value appropriated by the innovator, does not necessarily reduce the equilibrium threshold function $z$. In fact, increasing $\alpha$ not only increases the current value of the innovation but also the value of waiting for future increments in the value of the innovation. When the latter effect dominates, the result is a delay in innovation

16. This assumption is motivated by the observation that, while $R \& D$ results are often kept secret, the $R \& D$ investment, the size and facilities of the research labs, and the teams of researchers involved are usually public.

17. The patent race pay-offs may represent the innovation profit but can also represent the equilibrium profits of a competitive market game that takes place after the innovation is introduced into the market.

18. Our specification of the second-mover pay-off covers both instances where firms compete on the same research project, and instances where they compete on possibly very different projects. In the first case, the second-mover pay-off is determined only by the innovation patented by the first mover, who in a symmetric equilibrium is always ahead in the race. In the second case, each player's pay-off is determined by her own innovation.

19. This is consistent with the data of Hall, Jaffe and Trajtenberg (2001), who identify pharmaceutical and medical industries as the industries with the smallest patenting rate. Development costs are exceptionally high in pharmaceutical research because of the high costs of food and drug administration medical trials before a patented drug is allowed to be produced and marketed. 
disclosure. ${ }^{20}$ As it turns out, if the development cost $c_{0}$ is sufficiently large relative to the research cost, $c$, then stronger patent rights induce more frequent patenting. On the other hand, if research is sufficiently costly relative to development, then stronger patent rights reduce the patenting rate.

Proposition 1. In patent races, the equilibrium threshold function $z$ increases in the development cost $c_{0}$ and decreases in the research cost $c$. The relation between $z$ and $\alpha$, the fraction of innovation value appropriated by the innovator, is negative (positive) when $c$ is sufficiently small (large) relative to $c_{0}$, unless $\underline{u}$ is too large.

Further specializing our general results to patent races, we find that the patenting rate is smaller when innovation improvements are kept secret than when they are public information (Theorem 2). This result highlights a positive relationship between the patenting rate and industrial espionage, and may help explaining the acceleration in patenting rate, observed in the data since the mid 1980's (see Kortum and Lerner, 1999; Hall, Jaffe and Trajtenberg, 2001). ${ }^{21}$ It is well documented that the threat of economic and industrial espionage has been steadily growing, spurred on by technological change. The increasingly wide availability of wire-tapping and document reproduction technologies in the 1980's has been followed by the internet revolution of the 1990's, with all the implied threats to computer security. According to Lexicon Communications corporation, in 2005, economic espionage and the theft of trade secrets cost U.S. businesses more than $\$ 250$ billion and $\$ 1.2$ trillion over the last decade. ${ }^{22}$

Our result that private information counteracts preemption forces may also shed some light on the reported differences in citation numbers across industries. The National Bureau of Economic Research study by Hall, Jaffe and Trajtenberg (2001) on patent data sets identifies "old technology" industries, such as the mechanical and chemical ones, characterized by a large number of patents with few citations, and "new technology" industries such as computers and communications, characterized by a smaller number of patents but a larger number of citations. This dichotomy is consistent with our result that private information translates into a lower patenting rate but patents of higher economic value. In new, relatively unexplored technologies, different researchers may follow very different lines of research to achieve competing patentable innovations. As a result, they may be very uncertain about the prospects of the research conducted in competing labs. On the contrary, researchers are more likely to have a good shared knowledge of the possible incremental innovations achievable in old technologies, and therefore a good assessment of the prospects of the research conducted in competing labs.

Turning to welfare analysis, we compare the outcome when firms compete and keep their technological improvements secret, with the optimal choice of a team that aggregates the firms' information and maximizes the joint pay-off. Suppose that the value of the patented innovation does not effect the pay-off of the second mover and hence there are no competition externalities in the innovation's market. Theorem 3 implies that the team's patenting rate is lower than the

20. For simplicity, here we assume that once the innovation is patented, the patent race is over. The model can be extended so that a new patent race starts after the innovation is patented, with innovations building on the previously patented innovation. The result that increasing $\alpha$ may increase the threshold function $z$ holds as long as the current patent race competitors may be substituted by other firms in future races with sufficiently high probability. This assumption is motivated by the observation that, once an innovation is disclosed, the innovator may lose the technological leadership in the research field.

21. On a similar count, Hall and Ziedonis (2001), found that the number of patents per R\&D dollar in the semiconductor industry doubled between 1982 and 1992.

22. We see our explanation of the acceleration of the patenting rate as complementary and not as a substitute to the previous explanations that invoke a pro-patent legislative change in the mid 1980's and improved technological fertility. 
competing firms' rate, and that the team patents innovations of greater economic significance. Hence, secrecy is beneficial to competing firms as it brings the outcome closer to the collusive outcome.

\section{CONCLUSION}

We have presented a general analysis of preemption games with private information states increasing over time. The analysis initially appeared intractable because of the complexity of the agents' equilibrium updating of beliefs on the opponents' state. But we have calculated equilibria where each agent ends the game when her state is above a time-decreasing stopping threshold. We have compared these equilibria of our private information games with the equilibria of analogous preemption games with public information, and have found that the expected stopping time is shorter when information is public. We have studied the problem of a team aggregating the players' private information and maximizing the joint pay-off. As time grows large, competing players become willing to end the game at states for which the team planner would instruct them to continue. For the crucial case where the states are of private value, we have found that competing players stop the game too early relative to the collusive outcome.

We have applied these results to patent races, to find that secrecy slows down the speed of innovation disclosure. When there are no competition externalities in the innovation market, competing R\&D firms benefit from secrecy as it brings the equilibrium outcome closer to the collusive outcome. However, the collusive outcome is not achieved, as equilibrium durations are shorter than in the collusive outcome. Having calculated the closed form of the differential equation governing the equilibrium threshold, we have performed several comparative statics exercises. Surprisingly, we have found that strengthening patent rights may slow down innovation disclosure.

Our results can also be applied to innovation adoption and market entry games. Our analysis, available upon request, builds on the classic preemption game studied by Reinganum (1981) and later perfected by Fudenberg and Tirole (1985). In one application of that model, a process innovation is disclosed to competitors in a specific industry. When adopted, the innovation tilts the profits in favour of the first firm adopting the innovation; but adopting the new technology is costly. We expand the original model to posit adoption costs that depend on the stage of adaptation of the technology to the processes of each individual firm. Another application of the preemption game by Reinganum (1981) is a simple model of market entry. Competitors choose the timing of entry into a market. Entry is profitable and there is a first-mover advantage, but entering the market is costly. We suppose that each firm's entry costs depend on prior investment by the firm, e.g. in building capacity, in establishing a commercial network, in signing representation contracts, etc.

Assuming that our regularity conditions are satisfied, Theorem 2 implies that innovation adoption and market entry are delayed when the activities leading to innovation adoption and market entry are kept secret. As a result, secrecy stifles competition and is likely to reduce consumers' welfare. Consumers are likely to benefit from frequent market entry by new competitors and when production technologies are frequently improved. At the same time, Theorem $3 \mathrm{im}-$ plies that competing firms jointly benefit from secrecy, as it brings equilibrium durations closer to the collusive outcome. We also perform comparative statics analysis. We find that subsidizing innovation adoption and market entry makes them occur faster. However, subsidizing innovation development and investments leading to market entry delays innovation adoption and market entry. Intuitively, these subsidies are equivalent to reducing the waiting costs in our games and hence they induce longer expected stopping times. 


\section{APPENDIX A}

We begin the analysis by stating our results concerning the properties of $F(t, y) / F(t, z)$. In the interest of brevity, we omit their proofs that are available upon request from the authors.

Theorem A1. If the c.d.f. $G$ of the random increment $w$ is log-concave, i.e. the inverse hazard rate $g(w) / G(w)$ is weakly decreasing in $w$, then the c.d.f. $F(t, y) / F(t, z)$ is stochastically increasing in $t$ for any $z$ and $y \leq z$.

Theorem A2. If the increment c.d.f. $G(w)$ is differentiable, of connected support that includes $w=0$, then for any $z$, the c.d.f. $F(t, \cdot) / F(t, z)$ concentrates all mass on $z$ as $t$ goes to infinity, i.e.

$$
\lim _{t \rightarrow \infty} \frac{F(t, y)}{F(t, z)}=0 \text { for any } y<z \text {. }
$$

Proof of Theorem 1. We proceed in three Lemmas. We first show that, for any opponent's strategy, the best response of a player is a strategy $\sigma$ that can be represented as a time-dependent threshold function $z$.

Lemma A1. Under Conditions 1 and 2, for any $t$, and any strategy played by the opponent $j$, there is a unique threshold $z(t)$ such that continuing is optimal for player $i$ if $x<z(t)$, stopping is optimal if $x>z(t)$, and the optimal value $V^{i}(x, t)$ equals $u(x)$ at $x=z(t)$.

Proof. The proof proceeds in two steps. The first step shows that for any strategy played by the opponent, the unique best response of a player induces a value $V^{i}(x, t)$ continuous in $x$. Given continuity of $u$ and $\underline{u}$, the result is intuitive. Hence, we make its proof available only upon request.

Step 1. For any strategy played by player $j$, the best response of player $i$ induces a value $V^{i}(x, t)$ continuous in $x$.

The second step shows that, for any strategy played by the opponent $j$, if the best response by player $i$ induces a value $V^{i}(x, t)$ continuous in $x$, then it can be represented by a threshold function.

Step 2. Under Conditions 1 and 2, for any $t$, and any strategy played by the opponent, if the optimal value $V^{i}(x, y)$ is continuous in $x$, then there is a unique threshold $z(t)$ such that continuing is optimal for player $i$ if $x<z(t)$, stopping is optimal if $x>z(t)$, and $V^{i}(z(t), t)=u(z(t))$.

We want to show that for every $t$, there is a unique $z$ such that $u(x)<(=) V^{i}(x, t)$ if and only if $x<(\geq) z$. Since $V^{i}(x, t)$ is continuous in $x$, we only need to show that for any $t$ and any $x$ such that $u(x)<V^{i}(x, t)$,

$$
\lim _{x \rightarrow x^{\prime} \rightarrow 0} \sup \frac{V^{i}(x, t)-V^{i}\left(x^{\prime}, t\right)}{x-x^{\prime}}<u^{\prime}(x)
$$

because this implies that for any fixed $t$, the functions $V^{i}(x, t)$ and $u(x)$ can merge only once. Since $u^{\prime}(x)>0$, it follows that $u(x)$ is dominated by $V^{i}(x, t)$ if and only if $x$ is smaller than the merging point.

Consider a player $i$ and any pair $(x, t)$ such that the optimal strategy $\sigma$ dictates $\sigma(x, t)>t$. For any $x^{\prime}<x$ and $\left(x^{\prime}, t\right)$, let $V^{i}\left(\sigma^{\prime}, x^{\prime}, t\right)$ be the value associated with applying the same strategy $\sigma$ rescaled by a factor $x-x^{\prime}$, i.e. the strategy $\sigma^{\prime}$ such that $\sigma^{\prime}\left(x^{\prime \prime}+x^{\prime}, \tau\right)=\sigma\left(x^{\prime \prime}+x, \tau\right)$ for any $\tau \geq t$ and $x^{\prime \prime} \geq 0$. Since the optimal value at time $t$ and state $x^{\prime}$ is at least as large as the value $V^{i}\left(\sigma^{\prime}, x^{\prime}, t\right)$ of adopting the possibly suboptimal waiting strategy $\sigma^{\prime}$, we note that $V^{i}(x, t)-V^{i}\left(x^{\prime}, t\right) \leq V^{i}(x, t)-V^{i}\left(\sigma^{\prime}, x^{\prime}, t\right)$.

Fixing the opponent $j$ 's strategy $\sigma_{j}$ (which does not depend on $x$ ), the optimal pay-off at state $(x, t)$ is

$$
\begin{aligned}
V^{i}(x, t)= & \int E\left[u\left(x+\mathbf{x}\left(T_{i}\right)\right) \chi\left(T_{i}<T_{j}\right) e^{-r\left(T_{i}-t\right)}\right. \\
& \left.+\underline{u}\left(x+\mathbf{x}\left(T_{j}\right), y+\mathbf{y}\left(T_{j}\right)\right) \chi\left(T_{i}>T_{j}\right) e^{-r\left(T_{j}-t\right)}-\int_{t}^{\min \left\{T_{i}, T_{j}\right\}} c e^{-r v} d v \mid T_{j} \geq t\right] B\left(d y \mid T_{j} \geq t\right),
\end{aligned}
$$

and player $i$ 's expected pay-off for playing strategy $\sigma^{\prime}$ starting at state $\left(x^{\prime}, t\right)$ is

$$
\begin{aligned}
V^{i}\left(\sigma^{\prime}, x^{\prime}, t\right)= & \int E\left[u\left(x^{\prime}+\mathbf{x}\left(T_{i}^{\prime}\right)\right) \chi\left(T_{i}^{\prime}<T_{j}\right) e^{-r\left(T_{i}^{\prime}-t\right)}\right. \\
& \left.+\underline{u}\left(x^{\prime}+\mathbf{x}\left(T_{j}\right), y+\mathbf{y}\left(T_{j}\right)\right) \chi\left(T_{i}^{\prime}>T_{j}\right) e^{-r\left(T_{j}-t\right)}-\int_{t}^{\min \left\{T_{i}^{\prime}, T_{j}\right\}} c e^{-r v} d v \mid T_{j} \geq t\right] B\left(d y \mid T_{j} \geq t\right),
\end{aligned}
$$


where (i) the expectation $E$ is taken with respect to the sample paths $\mathbf{x}=\{x(\tau): \tau \geq t\}$ and $\mathbf{y}=\{y(\tau): \tau \geq t\}$, generated by our compound Poisson processes and such that $x(t)=0$ and $y(t)=0$; (ii) $T_{j}=\inf \left\{\tau: \sigma_{j}(y(\tau)+y, \tau)=\tau\right\}, T_{i}=$ $\inf \{\tau: \sigma(x(\tau)+x, \tau)=\tau\}$, and $T_{i}^{\prime}=\inf \left\{\tau: \sigma^{\prime}\left(x(\tau)+x^{\prime}, \tau\right)=\tau\right\}$; (iii) $\chi$ denotes the indicator function over sets of paths $(\mathbf{x}, \mathbf{y})$; and (iv) $B\left(y \mid T_{j} \geq t\right)=\operatorname{Pr}\left\{y(t) \leq t \mid T_{j} \geq t\right\}$ is the c.d.f. of the opponent state, $y(t)$, given that she has not left the game by time $t$.

Noting that $T_{i}^{\prime}=T_{i}$, and averaging across sample paths, $(\mathbf{x}, \mathbf{y})$, we obtain

$$
\begin{aligned}
\frac{V^{i}(x, t)-V^{i}\left(x^{\prime}, t\right)}{x-x^{\prime}} \leq & \frac{V^{i}(x, t)-V^{i}\left(\sigma^{\prime}, x^{\prime}, t\right)}{x-x^{\prime}} \\
= & \int E\left[\left(\frac{\underline{u}\left(x+\mathbf{x}\left(T_{j}\right), y+\mathbf{y}\left(T_{j}\right)\right)-\underline{u}\left(x^{\prime}+\mathbf{x}\left(T_{j}\right), y+\mathbf{y}\left(T_{j}\right)\right)}{x-x^{\prime}}\right) e^{-r\left(T_{j}-t\right)} \chi\left(T_{j}<T_{i}\right)\right. \\
& \left.+\left(\frac{u\left(x+\mathbf{x}\left(T_{i}\right)\right)-u\left(x^{\prime}+\mathbf{x}\left(T_{i}\right)\right)}{x-x^{\prime}}\right) e^{-r\left(T_{i}-t\right)} \chi\left(T_{i}<T_{j}\right) \mid T_{j} \geq t\right] B\left(d y \mid T_{j} \geq t\right) .
\end{aligned}
$$

Since,

$$
\begin{aligned}
& \lim _{x \rightarrow x^{\prime} \rightarrow 0}\left(\frac{u\left(x+\mathbf{x}\left(T_{i}\right)\right)-u\left(x^{\prime}+\mathbf{x}\left(T_{i}\right)\right)}{x-x^{\prime}}\right)=u^{\prime}\left(x+\mathbf{x}\left(T_{i}\right)\right), \\
& \lim _{x \rightarrow x^{\prime} \rightarrow 0}\left(\frac{\underline{u}\left(x+\mathbf{x}\left(T_{j}\right), y+\mathbf{y}\left(T_{j}\right)\right)-\underline{u}\left(x^{\prime}+\mathbf{x}\left(T_{j}\right), y+\mathbf{y}\left(T_{j}\right)\right)}{x-x^{\prime}}\right)=\underline{u}_{1}\left(x+\mathbf{x}\left(T_{j}\right), y+\mathbf{y}\left(T_{j}\right)\right),
\end{aligned}
$$

the Conditions $1, u^{\prime} \geq \underline{u}_{1}$, and $2, u^{\prime \prime} \leq 0$, yield:

$$
\begin{aligned}
\lim _{x \rightarrow x^{\prime} \rightarrow 0} \sup \frac{V^{i}(x, t)-V^{i}\left(x^{\prime}, t\right)}{x-x^{\prime}} \leq & \lim _{x \rightarrow x^{\prime} \rightarrow 0} \int E\left[\left(\frac{u(x)-u\left(x^{\prime}\right)}{x-x^{\prime}}\right) e^{-r\left(T_{i}-t\right)} \chi\left(T_{i}<T_{j}\right)\right. \\
& \left.+\left(\frac{u(x)-u\left(x^{\prime}\right)}{x-x^{\prime}}\right) e^{-r\left(T_{j}-t\right)} \chi\left(T_{j}<T_{i}\right) \mid T_{j} \geq\right] B\left(d y \mid T_{j} \geq t\right) \\
= & \lim _{x \rightarrow x^{\prime} \rightarrow 0} \int\left(\frac{u(x)-u\left(x^{\prime}\right)}{x-x^{\prime}}\right) E\left[e^{-r\left(T_{i}-t\right)} \chi\left(T_{i}<T_{j}\right)\right. \\
& \left.\quad+e^{-r\left(T_{j}-t\right)} \chi\left(T_{j}<T_{i}\right) \mid T_{j} \geq t\right] B\left(d y \mid T_{j} \geq t\right) .
\end{aligned}
$$

Since $E\left[T_{i}-t \mid T_{i}<T_{j}\right]>0, E\left[T_{j}-t \mid T_{j}<T_{i}\right]>0$, and $T_{i}$ and $T_{j}$ are independent of $x^{\prime}$, the quantity $E\left[e^{-r\left(T_{i}-t\right)}\right.$ $\left.\chi\left(T_{i}<T_{j}\right)+e^{-r\left(T_{j}-t\right)} \chi\left(T_{j}<T_{i}\right) \mid T_{j} \geq t\right]$ is strictly smaller than 1 and constant in $x^{\prime}$. Thus,

$$
\begin{aligned}
\lim _{x \rightarrow x^{\prime} \rightarrow 0} \sup \frac{V^{i}(x, t)-V^{i}\left(x^{\prime}, t\right)}{x-x^{\prime}} & <\lim _{x \rightarrow x^{\prime} \rightarrow 0} \int \frac{u(x)-u\left(x^{\prime}\right)}{x-x^{\prime}} B\left(d y \mid T_{j} \geq t\right) \\
& =\lim _{x \rightarrow x^{\prime} \rightarrow 0} \frac{u(x)-u\left(x^{\prime}\right)}{x-x^{\prime}}=u^{\prime}(x) .
\end{aligned}
$$

The limit for $x^{\prime}>x$ is similarly handled. The result that $u(z(t))=V^{i}(z(t), t)$ follows from continuity of $u(x)$ and $V^{i}(x, t)$.

The second lemma shows that there exists an admissible (i.e. strictly decreasing and defined on all the real line) solution to the differential equation (4). Further, it characterizes the limiting properties of all solutions to equation (4) for $t$ small and $t$ large.

Lemma A2. Under Conditions 1-4, there exists a strictly decreasing solution $z$ well defined on $\mathbb{R}_{+}$to the differential equation (4). Adding Conditions 5 and 6 , for any admissible solution $z$ to equation $(4), \lim _{z \rightarrow 0} z(t)=\bar{z}$ and $\lim _{t \rightarrow \infty} z(t)=\underline{z}$. 
Proof. We proceed in several steps.

Step 1. (Auxiliary Solution) For any $t$, the equation $\phi(t, z)=0$ has a solution $z$. The selection

$$
\hat{z}(t)=\min \{z: \phi(t, z)=0\},
$$

is strictly decreasing and satisfies $\hat{z}(0)=\bar{z}$ and $\lim _{t \rightarrow \infty} \hat{z}(t)=\underline{z}$.

For any $t$, Conditions 3 and 4 make sure that the function $\phi(t, z)$ is negative for $z$ small enough and positive for $z$ large enough. By continuity, $\phi$ has a zero $z$ for any $t$.

Since at time zero, the opponent's state is $y(0)=0$, and the probability of an increment arriving at time zero is zero, it must be that

$$
\frac{f(0, z)}{F(0, z)}=0 \text { for any } z>0
$$

It follows that equation $\phi(t, z)=0$ coincides with equation (5) for $t=0$.

By Theorem A2, $\lim _{t \rightarrow \infty} F(t, y) / F(t, z)=0$ for any $z>0$ and $y<z$. Hence, $\lim _{t \rightarrow \infty} \hat{z}(t)=\underline{z}$.

We finally want to show that the selection $\hat{z}$ is strictly decreasing in $t$. Because $\phi(t, z)$ crosses zero at $\hat{z}(t)$ from below, it follows that the selection $\hat{z}$ is decreasing if $\phi_{1}>0$. As $F(t, y) / F(t, z(t))$ increases in $t$ in first-order stochastic dominance by Theorem A1, it is sufficient to show that the expression $\rho \int_{z(t)-y}^{\infty}[u(z)-\underline{u}(z, y+w)] G(d w)$ increases in $y$. In fact,

$$
\begin{aligned}
& \frac{d}{d y} \rho \int_{z-y}^{\infty}[u(z)-\underline{u}(z, y+w)] G(d w) \\
& \quad=-\rho \int_{z-y}^{\infty} \underline{u}_{2}(z, y+w) G(d w)+\rho[u(z)-\underline{u}(z, z)] g(z-y)>0
\end{aligned}
$$

because $u>\underline{u}$ and $\underline{u}_{2} \leq 0$.

We note that $\hat{z}$ may be discontinuous at times $t$ such that $\phi_{2}(t, \hat{z}(t))=0$. Nevertheless, $\hat{z}$ is right continuous. Indeed, for any $t$, consider a sequence $\left\{t_{n}\right\}_{n \geq 1}$ such that $t<t_{n+1}<t_{n}$ for all $n$, and $\lim _{n \rightarrow+\infty} t_{n}=t$. Because $\hat{z}\left(t_{n}\right)$ is increasing and bounded above, it must converge and, by continuity of $\phi$, it must be that $\lim _{n \rightarrow+\infty} \hat{z}\left(t_{n}\right)=\hat{z}(t)$.

The next step establishes that, as long as $t$ is bounded away from zero, the ordinary differential equation (4) has a well-behaved solution field. Because the proof is entirely standard, it is omitted and made available upon request. Clearly, at $t=0$, the derivative $z^{\prime}(0)$ is indeterminate because $f(0, z) / F(0, z)=0$ for any $z>0$. We shall complete the solution at zero later in the paper.

Step 2. (Existence) For any small $\bar{\delta}>0$, consider the set $R(\bar{\delta})=\{(t, z): t \geq \bar{\delta}, z>0\}$. For any initial condition $\left(\delta, z_{\delta}\right) \in R(\bar{\delta})$, the ordinary differential equation (4) has a unique (twice-differentiable) solution path $z\left(t, \delta, z_{\delta}\right)$ defined on a set $\bar{R}(\bar{\delta}) \subseteq\{t: t \geq \bar{\delta}\}$, such that $z(\delta)=z_{\delta}$.

We are left to show that the ordinary differential equation (4) has an admissible solution, i.e. a solution strictly decreasing and well defined on $(0, \infty)$. Since step 2 allows for the possibility that solutions explode (i.e. have vertical asymptotes), we shall explicitly construct one admissible solution. We now show a result that will be useful in the construction.

Step 3. Consider any solution path $z(t)$ of the ordinary differential equation (4) defined on a set $\bar{R}(\bar{\delta})$. For any $T \in$ $\bar{R}(\bar{\delta})$, if $z(T) \leq \hat{z}(T)$, then $z(t)<\hat{z}(t)$, and hence $z^{\prime}(t)<0$, for all $t<T, t \in \bar{R}(\bar{\delta})$.

We first show that, for any $t \in \bar{R}(\bar{\delta}), \phi_{2}(t, \hat{z}(t))$ is bounded above. In fact, for any $(t, z)$,

$$
\begin{aligned}
\phi_{2}(t, z)= & r u^{\prime}(z)+c-\rho \int_{0}^{\infty}\left[u^{\prime}(z+w)-u^{\prime}(z)\right] G(d w) \\
& -\rho \int_{0}^{\infty}[\underline{u}(z, z+w)-u(z)] G(d w) \frac{F(t, z)}{F(t, z)} \\
& -\rho \int_{0}^{z} \frac{\partial}{\partial z}\left[\int_{z-y}^{\infty}[\underline{u}(z, y+w)-u(z)] G(d w) \frac{F(t, d y)}{F(t, z)}\right],
\end{aligned}
$$


and hence,

$$
\begin{aligned}
\phi_{2}(t, z)= & r u^{\prime}(z)+c-\rho \int_{0}^{\infty}\left[u^{\prime}(z+w)-u^{\prime}(z)\right] g(w) d w \\
& -\rho \int_{0}^{\infty}[\underline{u}(z, z+w)-u(z)] g(w) d w \\
& +\rho \frac{f(t, z)}{F(t, z)} \int_{0}^{z}\left[\int_{z-y}^{\infty}[\underline{u}(z, y+w)-u(z)] g(w) d w\right] \frac{f(t, y)}{F(t, z)} d y \\
& -\rho \int_{0}^{z}\left[\int_{z-y}^{\infty}\left[\underline{u}_{1}(z, y+w)-u^{\prime}(z)\right] g(w) d w\right] \frac{f(t, y)}{F(t, z)} d y \\
& +\rho \int_{0}^{z}[[\underline{u}(z, z)-u(z)] g(z-y)] \frac{f(t, y)}{F(t, z)} d y .
\end{aligned}
$$

Because $\underline{u}_{1}, u^{\prime}, g, u$, and $\underline{u}$ are bounded, the first four terms in the above expression are bounded above, and so are also all terms integrated against $f(t, y) / F(t, z)$. Further, note that for any finite $t \geq \bar{\delta}>0$, the term $f(t, y) / F(t, z)$ is finite for all $y \leq z$, and, likewise, $f(t, z) / F(t, z)$ is finite for all finite $t$ and $z>0$. This concludes that $\phi_{2}(t, \hat{z}(t))$ is bounded above for all $t \in \bar{R}(\bar{\delta})$.

Turning to prove the statement, suppose by contradiction that $z(t) \geq \hat{z}(t)$ for some $t<T$.

Note that, without loss of generality, we can take $z(t)>\hat{z}(t)$ because if $z(t)=\hat{z}(t)$ for some $t<T$, then $z\left(t^{\prime}\right)>\hat{z}\left(t^{\prime}\right)$ for all $t^{\prime}$ on a right neighbourhood of $t$. In fact, because $z(t)=\hat{z}(t)$, it follows that $z^{\prime}(t)=0$. Because $\hat{z}$ is strictly decreasing and $\phi_{2}(t, \hat{z}(t))$ is bounded above, there exists $\alpha>0$ such that for all small positive $h, \hat{z}(\tau+h)-\hat{z}(\tau)<-\alpha h$. Combining this with $z^{\prime}(\tau)=0$ yields $z(\tau+h)>\hat{z}(\tau+h)$.

Because $\hat{z}$ is strictly decreasing, $z(t)>\hat{z}(t)>\hat{z}(T) \geq z(T)$. Since $z$ is continuous and continuously differentiable, and $z(t)>z(T)$, there must exist a time $\tau \in(t, T)$ such that $z(\tau)=\hat{z}(t)$ and $z^{\prime}(\tau) \leq 0$. But by definition $\phi(t, \hat{z}(t))=$ 0 . Because $\tau>t$, and because we have proved in Step 1 that $\phi_{1}(t, z)>0$ for all $(t, z)$, it follows that $\phi(\tau, \hat{z}(t))=$ $\phi(\tau, z(\tau))>0$, and hence that $z^{\prime}(\tau)>0$. We have obtained a contradiction.

Step 4. (Admissibility) The ordinary differential equation (4) has a strictly decreasing solution path $z(t)$ defined on $(0, \infty)$.

Fix a time $\delta>0$. We first construct an admissible solution in the range $[\delta, \infty)$. For any $T>\delta$, consider the initial condition $\left(\delta, z_{\delta, T}\right)$ identifying the solution path $z\left(t, \delta, z_{\delta, T}\right)$ such that $z\left(T, \delta, z_{\delta, T}\right)=\hat{z}(T)$. First, note that, by Step 3 , $z\left(t, \delta, z_{\delta, T}\right)<\hat{z}(t)$ for all $t \in[\delta, T)$. Further, by Step 2 , for any point $(z, t)$ with $t \geq \delta$ and $z>0$, the solution path $z(t)$ such that $z(t)=z$ is unique. Hence, for any $T^{\prime}>T$, the solution path $z(t, \delta, z \delta, T)$ must lie above the solution $z\left(t, \delta, z_{\delta, T^{\prime}}\right)$. For any $T$, we proceed analogously to identify the initial condition $\left(\delta, \underline{z}_{\delta, T}\right)$ pinning down the solution path $z(t, \delta, \underline{z} \delta, T)$ such that $z\left(T, \delta, \underline{z}_{\delta, T}\right)=\underline{z}$. The state $z_{\delta, T}$ decreases in $T$, whereas the state $\underline{z}_{\delta, T}$ increases in $T$. Furthermore, $\underline{z}_{\delta, T}<z_{\delta, T}$ because $\hat{z}(T)>\underline{z}$ for any $T$. Hence, there exists $z \delta$ such that $\lim _{T \rightarrow+\infty} \underline{z} \delta, T \leq z \delta \leq \lim _{T \rightarrow+\infty} z_{\delta, T}$. By construction, the solution path $\bar{z}\left(t, \delta, z_{\delta}\right)$ is such that $z\left(t, \delta, z_{\delta}\right)<\hat{z}(t)$ on the whole range $t \geq \delta$. Hence, $z\left(t, \delta, z_{\delta}\right)$ strictly decreases in $t$ for all $t \geq \delta$. Note also $\lim _{t \rightarrow \infty} z(t, \delta, z \delta) \geq \underline{z}$, and that $z_{\delta}$ need not be unique.

We now complete the admissible solution $z\left(\cdot, \delta, z_{\delta}\right)$ to the left of $\delta$. Consider a strictly decreasing sequence $\left\{\delta_{n}\right\}_{n \geq 1}$ such that $\delta_{1}=\delta$ and $\delta_{n} \rightarrow 0$. Let $z_{1}=z_{\delta}$, and, for any $n$, define $z_{n+1}$ recursively by solving the condition $z\left(\delta_{n}, \delta_{n+1}, z_{n+1}\right)=z_{n}$. For all $n$, Step 2 implies that $z_{n}$ is well defined and that the function $z\left(\cdot, \delta_{n+1}, z_{n+1}\right)$ is (twice) differentiable on $\left[\delta_{n+1}, \delta_{n}\right)$. By construction, $z\left(t, \delta_{n+1}, z_{n+1}\right)=z\left(t, \delta_{n}, z_{n}\right)$ for all $t \geq \delta_{n}$.

We now show by induction that, for all $n, z\left(t, \delta_{n+1}, z_{n+1}\right)<\hat{z}(t)$ for all $t \in\left[\delta_{n+1}, \delta_{n}\right]$. In fact, we have previously shown that $z\left(\delta_{1}, \delta_{1}, z_{1}\right)<\hat{z}\left(\delta_{1}\right)$. Further, by the induction hypothesis, $z\left(\delta_{n}, \delta_{n}, z_{n}\right)<\hat{z}\left(\delta_{n}\right)$. Because $z\left(t, \delta_{n+1}, z_{n+1}\right)=$ $z\left(t, \delta_{n}, z_{n}\right)$ for all $t \geq \delta_{n}$, it follows that $z\left(\delta_{n}, \delta_{n+1}, z_{n+1}\right)<\hat{z}\left(\delta_{n}\right)$. By Step 3, it follows that $z\left(t, \delta_{n+1}, z_{n+1}\right)<\hat{z}(t)$ for all $t \in\left[\delta_{n+1}, \delta_{n}\right)$. This concludes the proof of the induction argument.

We have shown that $\hat{z}\left(\delta_{n+1}\right)>z_{n+1}$ and that for any $n, z\left(t, \delta_{n+1}, z_{n+1}\right)$ is strictly decreasing in $t$ for all $t \geq \delta_{n+1}$. As a result, for any $n, \hat{z}(0)>\hat{z}\left(\delta_{n+1}\right)>z_{n+1}>z_{n}$. Because, the sequence $\left\{z_{n}\right\}$ is strictly increasing and bounded, it admits a limit for $n \rightarrow \infty$. Hence, by taking the limit for $n \rightarrow \infty$, we have completed the solution $z\left(t, \delta, z_{\delta}\right)$ on the interval $(0, \delta)$, and proved that the completed solution $z(t)$ is strictly decreasing. Note also that we have shown that $\lim _{t \rightarrow 0} z(t) \leq \bar{z}$.

The above steps of the proof have proved existence of an admissible solution of the ordinary differential equation (4). We now characterize all admissible solutions $t \rightarrow 0$. 
Step 5. (Solution Characterization for $t \rightarrow 0$ ) For any admissible (i.e. decreasing and well defined) solution path $z(t)$ of the ordinary differential equation (4),

$$
\lim _{t \rightarrow 0^{+}} \frac{f(t, z(t))}{F(t, z(t))} z^{\prime}(t)=0, \text { and hence } \lim _{t \rightarrow 0} z(t)=\bar{z}
$$

We inspect again the ordinary differential equation (4). Because, as already pointed out, $f(0, z) / F(0, z)=0$ for any $z>0$, it follows that (i) the derivative $z^{\prime}(t)$ is indeterminate at $t=0$ and (ii) $\lim _{t \rightarrow 0^{+}} z(t)$ is a solution of equation (5) unless

$$
\lim _{t \rightarrow 0^{+}} \frac{f(t, z(t))}{F(t, z(t))} z^{\prime}(t)=K<0
$$

where $K$ is a negative constant. By the properties of Poisson arrival,

$$
\lim _{t \rightarrow 0^{+}} \frac{f(t, z(t))}{F(t, z(t))} z^{\prime}(t)=\lim _{t \rightarrow 0^{+}} \frac{\rho \operatorname{tg}(z(t))}{(1-\rho t)+\rho t G(z(t))} z^{\prime}(t)=\lim _{t \rightarrow 0^{+}} \rho \operatorname{tg}(z(t)) z^{\prime}(t) .
$$

Note that $\lim _{t \rightarrow 0^{+}} \rho \operatorname{tg}(z(t)) z^{\prime}(t)=K$ requires that $z^{\prime}(t)=k / t+o(1 / t)$, where $k$ is a negative constant and $o(1 / t)$ denotes a term that converges to zero if multiplied by $t$ when $t \rightarrow 0$; hence it requires that $\lim _{t \rightarrow 0^{+}} z(t)=\lim _{t \rightarrow 0^{+}} k \log t=$ $+\infty$. Condition 4 implies that $\phi(t, z(t))>0$, and hence $z^{\prime}(t)>0$, for $z(t)$ large enough. This contradicts $z^{\prime}(t)<0$. The above result implies that $\lim _{t \rightarrow 0^{+}} z(t)$ is a solution of equation (5). To conclude, note that any admissible solution $z(t)$ of the ordinary differential equation (4) cannot cross the function $\hat{z}$ or else it is increasing over a non-degenerate interval (by Step 3). This implies that $\lim _{t \rightarrow 0^{+}} z(t) \leq \lim _{t \rightarrow 0^{+}} \hat{z}(t)=\bar{z}$, and hence by the previous arguments that $\lim _{t \rightarrow 0^{+}} z(t)=\bar{z}$.

The next result characterizes all admissible solutions $z(t)$ for $t \rightarrow \infty$ under conditions $1-6$.

Step 6. (Solution Characterization for $t \rightarrow \infty$ ) Under Conditions 1-6, for any admissible (i.e. decreasing and well defined) solution path $z(t)$ of the ordinary differential equation (4),

$$
\lim _{t \rightarrow+\infty} \frac{f(t, z(t))}{F(t, z(t))} z^{\prime}(t)=0 \text { and hence } \lim _{t \rightarrow \infty} z(t)=\underline{z}
$$

Consider the expression:

$$
\underline{\phi}(z)=(r+2 \rho) u(z)+c-\rho \int_{0}^{\infty}[u(z+w)+\underline{u}(z, z+w)] G(d w)
$$

Since $\underline{u}_{2} \leq 0, u^{\prime} \geq \underline{u}_{1}$ (Condition 1), $u^{\prime \prime} \leq 0$ (Condition 2), $\underline{u}_{22} \leq 0$ (Condition 5), and $\underline{u}_{12} \leq 0$ (Condition 6), the expression $\underline{\phi}(z)$ strictly increases in $z$ as

$$
\begin{aligned}
\underline{\phi}^{\prime}(z)= & r u^{\prime}(z)+\rho \int_{0}^{\infty}\left[u^{\prime}(z)-u^{\prime}(z+w)+u^{\prime}(z)-\underline{u}_{1}(z, z)+\underline{u}_{1}(z, z)-\underline{u}_{1}(z, z+w)\right. \\
& \left.-\underline{u}_{2}(z, z)+\underline{u}_{2}(z, z)-\underline{u}_{2}(z, z+w)\right] G(d w)>0 .
\end{aligned}
$$

Hence, there is a unique solution to equation (6). So, for any admissible solution $z$ of the ordinary differential equation (4), it must be the case that $\lim _{t \rightarrow \infty} z(t) \leq \underline{z}$ or else $\phi(t, z(t))>0$ and hence $z^{\prime}(t)>0$ for $t$ sufficiently large. Further, because $z(t)$ is strictly decreasing and bounded below, as $z(t) \geq 0$, it must be the case that $z(t)$ converges to a limit as $t \rightarrow \infty$. Suppose by contradiction, that $\lim _{t \rightarrow \infty} z(t)<\underline{z}$. By inspecting the ordinary differential equation (4), this implies that $z^{\prime}(t) f(t, z(t)) / F(t, z(t))$ converges to a real valued limit $-K<0$ as $t \rightarrow \infty$. By using L'Hopital's rule, we obtain

$$
\begin{aligned}
\lim _{t \rightarrow \infty} \frac{f(t, z(t)) z^{\prime}(t)}{F(t, z(t))}=\lim _{t \rightarrow \infty} \frac{f_{1}(t, z(t)) z^{\prime}(t)+f_{2}(t, z(t))\left(z^{\prime}(t)\right)^{2}+f(t, z(t)) z^{\prime \prime}(t)}{F_{1}(t, z(t))+F_{2}(t, z(t)) z^{\prime}(t)} \\
\quad=\lim _{t \rightarrow \infty} \frac{\frac{\rho \int_{0}^{z(t)}[f(t, z(t)-w)-f(t, z(t))] G(d w)}{F(t, z(t))} z^{\prime}(t)-\frac{\rho F(t, z(t)) g(z(t))}{F(t, z(t))} z^{\prime}(t)+\frac{f_{2}(t, z(t))}{F(t, z(t))}\left(z^{\prime}(t)\right)^{2}+\frac{f(t, z(t)) z^{\prime}(t)}{F(t, z(t))} \frac{z^{\prime \prime}(t)}{z^{\prime}(t)}}{\frac{\rho \int_{0}^{z(t)}[F(t, z(t)-w)-F(t, z(t))] G(d w)}{F(t, z(t))}+\frac{f(t, z(t)) z^{\prime}(t)}{F(t, z(t))}} .
\end{aligned}
$$




\section{HOPENHAYN \& SQUINTANI PREEMPTION GAMES}

where the second equality follows by the formula for $F_{1}(t, x)$ and by

$$
\begin{aligned}
f_{1}(t, x) & =F_{12}(t, x)=\rho[F(t, 0)-F(t, x)] g(x)+\rho \int_{0}^{x}[f(t, x-w)-f(t, x)] G(d w) \\
& =\rho \int_{0}^{x}[f(t, x-w)-f(t, x)] G(d w)-\rho F(t, x) g(x) .
\end{aligned}
$$

From Theorem A2,

$$
\lim _{t \rightarrow \infty} \frac{F(t, x)}{F(t, z(t))}=0 \text { for all } x<z(t),
$$

hence,

$$
\begin{aligned}
& \lim _{t \rightarrow \infty} \frac{\rho \int_{0}^{z(t)}[F(t, z(t)-w)-F(t, z(t))] G(d w)}{F(t, z(t))} \\
&=\lim _{t \rightarrow \infty} \rho \int_{0}^{z(t)} \frac{F(t, z(t)-w)}{F(t, z(t))} G(d w)-\rho \int_{0}^{z(t)} \frac{F(t, z(t))}{F(t, z(t))} G(d w)=-\lim _{t \rightarrow \infty} \rho G(z(t)) . \\
& \lim _{t \rightarrow \infty} \frac{\rho \int_{0}^{z(t)}[f(t, z(t)-w)-f(t, z(t))] G(d w)}{F(t, z(t))} \\
&= \lim _{t \rightarrow \infty}\left(\rho \int_{0}^{z(t)} \frac{f(t, z(t)-w)}{F(t, z(t))} G(d w)-\rho \frac{f(t, z(t))}{F(t, z(t))} G(z(t))\right) \\
& \leq \lim _{t \rightarrow \infty}\left(\rho \sup _{w} g(w) \int_{0}^{z(t)} \frac{f(t, z(t)-w)}{F(t, z(t))} d w-\rho \frac{f(t, z(t))}{F(t, z(t))} G(z(t))\right) \\
&= \lim _{t \rightarrow \infty}\left(\rho \sup _{w} g(w) \int_{0}^{z(t)} \frac{f(t, \hat{w})}{F(t, z(t))} d \hat{w}-\rho \frac{f(t, z(t))}{F(t, z(t))} G(z(t))\right) \\
&=\rho \sup _{w} g(w)-\rho \lim _{t \rightarrow \infty} \frac{f(t, z(t))}{F(t, z(t))} G(z(t)) .
\end{aligned}
$$

Due to Theorem A2, we have that $\lim _{t \rightarrow \infty} f_{2}(t, z(t)) / F(t, z(t))>0$. Since $z^{\prime \prime}(t) / z^{\prime}(t)=\frac{d}{d t} \ln \left(-z^{\prime}(t)\right)$ and $\lim _{t \rightarrow \infty}\left(-z^{\prime}(t)\right)=0^{+}$, we have that $\lim _{t \rightarrow \infty} z^{\prime \prime}(t) / z^{\prime}(t)=-\infty$. Hence, we obtain a contradiction, as:

$$
\begin{aligned}
-K & =\lim _{t \rightarrow \infty} \frac{f(t, z(t)) z^{\prime}(t)}{F(t, z(t))} \\
& \leq \lim _{t \rightarrow \infty} \frac{\rho \sup _{w} g(w) z^{\prime}(t)+\rho G(z(t)) K-\rho g(z(t)) z^{\prime}(t)+\frac{f_{2}(t, z(t))}{F(t, z(t))}\left(z^{\prime}(t)\right)^{2}-K \frac{z^{\prime \prime}(t)}{z^{\prime}(t)}}{-\rho G(z(t))-K}=-\infty .
\end{aligned}
$$

This conclude the proof of the second part of Lemma A.2. \|

The proof of Theorem 1 is concluded by establishing that the equilibrium value $V(x, t)$ is differentiable in $t$.

Lemma A3. If both players play according to a strictly decreasing continuously differentiable threshold function $z(t)$, then the equilibrium value $V(x, t)$ is differentiable in $t$.

We establish this result by forward-calculating $V(x, t)$ when both players play according to a strictly decreasing continuously differentiable threshold function $z(t)$, and then by inspecting the resulting formula for $V(x, t)$. Because the argument is calculation incentive and the calculations are not further used in the analysis, we make the proof available only upon request. $\|$

Proof of Theorem 2. We proceed in four steps.

For any equilibrium $\sigma$, the first step derives an upper bound to the set $C(\sigma)$. Intuitively, each player $i$ must necessarily stop the game for any $x \geq \check{z}$, where $\check{z}$ is the unique solution of the equation

$$
r u(\check{z})=-c+\rho \int_{0}^{\infty}[u(\check{z}+w)-u(\check{z})] G(d w) .
$$


In fact, this equation describes the flow costs and benefits when player $i$ acts as a "monopolist" and there are no costs of waiting induced by competition with the opponent. As this step is intuitive and is not central to the argument in the proof, it is omitted and made available upon request.

Step 1. For any $\sigma$, the $\operatorname{set} C(\sigma)$ is a subset of $\left\{\left(x_{A}, x_{B}\right): x_{A} \leq \check{z}, x_{B} \leq \check{z}\right\}$.

For any equilibrium $\sigma$, the second step of the proof determines the marginal incentives to stop the game on the "north-east" frontier of $C(\sigma)$.

Step 2. For any equilibrium $\sigma$, consider any pair $\left(x_{A}, x_{B}\right)$ that belongs to $\bar{C}(\sigma)$, the closure of $C(\sigma)$, such that $\left(x_{A}+w\right) \notin C(\sigma)$ and $\left(x_{B}+w\right) \notin C(\sigma)$ for all $w>0$. For any $i=A, B$,

$$
\tilde{\phi}\left(x_{i}, x_{j}\right) \equiv(r+2 \rho) u\left(x_{i}\right)+c-\rho \int_{0}^{\infty}\left[\bar{u}\left(x_{i}+w, x_{j}\right)+\bar{u}\left(x_{i}, x_{j}+w\right)\right] G(d w) \leq 0 .
$$

For any $\delta>0$, there is a pair $\left(x_{A}^{\delta}, x_{B}^{\delta}\right) \in C(\sigma)$ such that $\left|x_{A}^{\delta}-x_{A}\right|<\delta$, and $\left|x_{B}^{\delta}-x_{B}\right|<\delta$, possibly $\left(x_{A}^{\delta}, x_{B}^{\delta}\right)=$ $\left(x_{A}, x_{B}\right)$. Since $\left(x_{A}^{\delta}, x_{B}^{\delta}\right) \in C(\sigma)$, we know that for any $i$,

$$
\begin{aligned}
u\left(x_{i}^{\delta}\right) \leq & V^{i}\left(x_{i}^{\delta}, x_{j}^{\delta}\right) \\
= & \frac{1}{r+2 \rho}\left\{-c+\rho \int_{0}^{\left|x_{i}-x_{i}^{\delta}\right|}\left[V^{i}\left(x_{i}^{\delta}+w, x_{j}^{\delta}\right)+V^{i}\left(x_{i}^{\delta}, x_{j}^{\delta}+w\right)\right] G(d w)\right. \\
& \left.+\rho \int_{\left|x_{i}-x_{i}^{\delta}\right|}^{\infty}\left[\bar{u}\left(x_{i}^{\delta}+w, x_{j}^{\delta}\right)+\bar{u}\left(x_{i}^{\delta}, x_{j}^{\delta}+w\right)\right] G(d w)\right\} .
\end{aligned}
$$

Exploiting the boundedness of $\bar{u}$ and $V^{i}$, we obtain

$$
u\left(x_{i}\right) \leq V^{i}\left(x_{i}, x_{j}\right)=\frac{1}{r+2 \rho}\left\{-c+\rho \int_{0}^{\infty}\left[\bar{u}\left(x_{i}+w, x_{j}\right)+\bar{u}\left(x_{i}, x_{j}+w\right)\right] G(d w)\right\} .
$$

The proof follows by rearranging this inequality.

Step 3. For any equilibrium $\sigma$, the set $C(\sigma)$ is contained in $\left\{\left(x_{A}, x_{B}\right): x_{A}+x_{B} \leq 2 \underline{z}\right\}$.

For any $y$, let $\tilde{z}(y)$ be the unique $z$ that solves $\tilde{\phi}(z, y)=0$. The threshold $\tilde{z}$ is well defined because

$$
\begin{aligned}
\frac{d \tilde{\phi}(z, y)}{d z} & =r u^{\prime}(z)+\rho \int_{0}^{\infty}\left[u^{\prime}(z)-\bar{u}_{1}(z+w, y)+u^{\prime}(z)-\bar{u}_{1}(z, y+w)\right] G(d w) \\
& \geq r u^{\prime}(z)+\rho \int_{0}^{\infty}\left[u^{\prime}(z)-u^{\prime}(z+w)+u^{\prime}(z)-u^{\prime}(z)\right] G(d w)>0
\end{aligned}
$$

where the first inequality follows from Condition $1, u>\bar{u}_{1}$, and the second from Condition $2, u^{\prime \prime} \leq 0$.

To compare $\underline{z}$ with the state $\tilde{z}=\sup \{z: z=\tilde{z}(z)\}$, note that

$$
\begin{aligned}
\underline{\phi}(\tilde{z}) & =\underline{\phi}(\tilde{z})-\tilde{\phi}(\tilde{z}, \tilde{z}) \\
& =-\rho \int_{0}^{\infty}[u(\tilde{z}+w)+\underline{u}(\tilde{z}, \tilde{z}+w)-\bar{u}(\tilde{z}+w, \tilde{z})-\bar{u}(\tilde{z}, \tilde{z}+w)] G(d w) \\
& =-\frac{\rho}{2} \int_{0}^{\infty}[u(\tilde{z}+w)+\underline{u}(\tilde{z}, \tilde{z}+w)-\underline{u}(\tilde{z}+w, \tilde{z})-u(\tilde{z})] G(d w) \\
& =-\frac{\rho}{2} \int_{0}^{\infty}[u(\tilde{z}+w)-u(\tilde{z})+\underline{u}(\tilde{z}, \tilde{z}+w)-\underline{u}(\tilde{z}, \tilde{z})-\underline{u}(\tilde{z}+w, \tilde{z})+\underline{u}(\tilde{z}, \tilde{z})] G(d w) \leq 0
\end{aligned}
$$

under Condition $8, u^{\prime}(x)+\underline{u}_{2}(y, x)-\underline{u}_{1}(x, y) \geq 0$. This implies that $\underline{z} \geq \tilde{z}$ because $\underline{\phi}(z)$ is strictly increasing in $z$ under Conditions $1,2,5$, and 6 . 


\section{HOPENHAYN \& SQUINTANI PREEMPTION GAMES}

We now show that $d \tilde{z}(y) / d y>-1$,

$$
\begin{aligned}
& \frac{d \tilde{z}(y)}{d y}+1=-\frac{\tilde{\phi}_{2}(z, y)}{\tilde{\phi}_{1}(z, y)}+1 \\
\propto & (r+2 \rho) u^{\prime}(z)-\rho \int_{0}^{\infty}\left[\bar{u}_{1}(z+w, y)+\bar{u}_{1}(z, y+w)-\bar{u}_{2}(z+w, y)-\bar{u}_{2}(z, y+w)\right] G(d w) \\
= & (r+2 \rho) u^{\prime}(z)-\frac{\rho}{2} \int_{0}^{\infty}\left[u^{\prime}(z+w)+\underline{u}_{1}(z+w, y)-\underline{u}_{2}(z+w, y)\right. \\
& \left.+u^{\prime}(z)+\underline{u}_{1}(z, y+w)-\underline{u}_{2}(z, y+w)\right] G(d w) \\
\geq & (r+2 \rho) u^{\prime}(z)-\rho \int_{0}^{\infty}\left[u^{\prime}(z+w)+u^{\prime}(z)\right] G(d w)>0 .
\end{aligned}
$$

The first inequality follows from Condition $7, u^{\prime}(x) \geq \underline{u}_{1}(x, y)-\underline{u}_{2}(x, y)$. The second inequality follows because $u^{\prime}>0$ and $u^{\prime \prime} \leq 0$ (Condition 2).

As $\underline{z} \geq \tilde{z}$ and $d \tilde{z}(y) / d y>-1$, we conclude that for all $x_{A}+x_{B} \geq 2 \underline{z}, x_{A} \neq x_{B}$, it is either the case that $\tilde{\phi}\left(x_{A}, x_{B}\right)>$ 0 , when $x_{A}>x_{B}$ or that $\tilde{\phi}\left(x_{B}, x_{A}\right)>0$, when $x_{B}>x_{A}$. It follows that $\left(x_{A}, x_{B}\right) \notin C(\sigma)$ for all $x_{A}+x_{B} \geq 2 \underline{z}, x_{A} \neq$ $x_{B}$. Suppose to the contrary that $\left(x_{A}, x_{B}\right) \in \bar{C}(\sigma)$ and $x_{A}+x_{B} \geq 2 z, x_{A} \neq x_{B}$. Since $C(\sigma)$ is bounded, there exist $\left(\bar{x}_{A}, \bar{x}_{B}\right) \in \bar{C}(\sigma), \bar{x}_{A} \geq x_{A}$ and $\bar{x}_{B} \geq x_{B}$, such that $\left(\bar{x}_{A}+w\right) \notin C(\sigma)$ and $\left(\bar{x}_{B}+w\right) \notin C(\sigma)$ for all $w>0$. Because $\bar{x}_{A}+\bar{x}_{B} \geq 2 \underline{z}$, it follows that $\tilde{\phi}\left(\bar{x}_{A}, \bar{x}_{B}\right)>0$ or $\tilde{\phi}\left(\bar{x}_{B}, \bar{x}_{A}\right)>0$ or both, contradicting Step 2 .

Step 4. For any equilibrium $\sigma, E\left[\inf \left\{\tau:\left(x_{A}(\tau), x_{B}(\tau)\right) \notin C(\sigma)\right\}\right]<E\left[\inf \left\{\tau: x_{A}(\tau) \geq \underline{z}\right.\right.$ or $\left.\left.x_{B}(\tau) \geq \underline{z}\right\}\right]$.

As $x_{A}(t)$ and $x_{B}(t)$ are independent $E\left[\inf \left\{\tau: x_{A}(\tau) \geq \underline{z}\right.\right.$ or $\left.\left.x_{B}(\tau) \geq \underline{z}\right\}\right]=E\left[\inf \left\{\tau: x_{A}(\tau) \geq \underline{z}\right\}\right]$. The distribution of the process $q(t)=x_{A}(t)+x_{B}(t)$ belongs to the same class as either $x_{A}(t)$ or $x_{B}(t)$, but with a Poisson arrival rate of $2 \rho$ instead of $\rho$. Due to the properties of Poisson processes, the law of $s(t)=q(t / 2)=x_{A}(t / 2)+x_{B}(t / 2)$ is the same as the law of either $x_{A}(t)$ or $x_{B}(t)$. As $x_{A}(\tau)=x_{B}(\tau)$ occurs with probability zero, it follows that

$$
\begin{aligned}
& E\left[\inf \left\{\tau:\left(x_{A}(\tau), x_{B}(\tau)\right) \notin C(\sigma)\right\}\right]<E\left[\inf \left\{\tau: x_{A}(\tau)+x_{B}(\tau) \geq 2 \underline{z}\right\}\right]= \\
& =E\left[\inf \left\{\tau: x_{A}(\tau / 2)+x_{B}(\tau / 2) \geq \underline{z}\right\}\right]=E\left[\inf \left\{\tau: x_{A}(\tau) \geq \underline{z}\right\}\right]=E\left[\inf \left\{\tau: x_{A}(\tau) \geq \underline{z} \text { or } x_{B}(\tau) \geq \underline{z}\right\}\right] .
\end{aligned}
$$

The proof of Lemma 2 is analogous to the proof of Lemma A.1. Hence, we omit it. It is available upon request.

Proof of Theorem 3. As the stopping boundary $z^{*}$ is strictly decreasing and symmetric, it suffices to compare the state $\bar{x}$ such that $\bar{x}=z^{*}(\bar{x})$ with the thresholds $\underline{z}$ and $\bar{z}$.

When $\bar{x}=z^{*}(\bar{x})$, equation (9) can be written as

$$
\hat{\phi}(\bar{x}) \equiv(r+2 \rho) \frac{u(\bar{x})+\underline{u}(\bar{x}, \bar{x})}{2}+c-\rho \int_{0}^{\infty}[u(\bar{x}+w)+\underline{u}(\bar{x}, \bar{x}+w)] G(d w)=0
$$

because $u^{*}(\bar{x}+w, \bar{x})=u^{*}(\bar{x}, \bar{x}+w)=u(\bar{x}+w)+\underline{u}(\bar{x}, \bar{x}+w)$.

We now subtract equation (6) from equation (A.2), calculated at $\underline{z}$ to obtain

$$
\hat{\phi}(\underline{z})=\hat{\phi}(\underline{z})-\underline{\phi}(\underline{z})=(r+2 \rho)\left[\frac{\underline{u}(\underline{z}, \underline{z})-u(\underline{z})}{2}\right]<0
$$

because $u(\underline{z})>\underline{u}(\underline{z}, \underline{z})$. To conclude that $\underline{z}<\bar{x}$, we only need to establish that for all $x$,

$$
\begin{aligned}
\hat{\phi}^{\prime}(x)= & (r+2 \rho) \frac{u^{\prime}(x)+\underline{u}_{1}(x, x)+\underline{u}_{2}(x, x)}{2}+c \\
& -\rho \int_{0}^{\infty}\left[u^{\prime}(x+w)+\underline{u}_{1}(x, x+w)+\underline{u}_{2}(x, x+w)\right] G(d w)>0,
\end{aligned}
$$

which follows from $\underline{u}_{1} \geq 0, u^{\prime}+\underline{u}_{2}>0$ (Condition 9), $u^{\prime \prime} \leq 0$ (Condition 2$), \underline{u}_{2} \leq 0$ (Condition 5), and $\underline{u}_{12} \leq 0$ (Condition 6). 
We now compare $\bar{x}$ with the threshold $\bar{z}$. When $\underline{u}_{2}=0$, dropping the dependence of $\underline{u}$ in the second argument, the quantities $\bar{z}$ and $\bar{x}$, respectively, solve

$$
\bar{\phi}(\bar{z}) \equiv(r+\rho) u(\bar{z})+c-\rho \int_{0}^{\infty} u(\bar{z}+w) G(d w)-\rho[1-G(\bar{z})][\underline{u}(\bar{z})-u(\bar{z})]=0
$$

and

$$
\hat{\phi}(\bar{x})=(r+2 \rho) \frac{u(\bar{x})+\underline{u}(\bar{x})}{2}+c-\rho \int_{0}^{\infty} u(\bar{x}+w) G(d w)-\rho \underline{u}(\bar{x})=0 .
$$

As $u^{\prime}>0, \underline{u}^{\prime}>0$, and $u^{\prime \prime} \leq 0$ (Condition 2), it follows that $\hat{\phi}^{\prime}(x)>0$ for all $x$. Hence, we only need to show that

$$
\begin{aligned}
\hat{\phi}(\bar{z}) & =\hat{\phi}(\bar{z})-\bar{\phi}(\bar{z})=(r+2 \rho) \frac{u(\bar{z})+\underline{u}(\bar{z})}{2}-\rho \underline{u}(\bar{z})-\{(r+\rho) u(\bar{z})-\rho[1-G(\bar{z})][\underline{u}(\bar{z})-u(\bar{z})]\} \\
& =r \frac{\underline{u}(\bar{z})-u(\bar{z})}{2}+\rho[1-G(\bar{z})][\underline{u}(\bar{z})-u(\bar{z})]<0,
\end{aligned}
$$

which follows because $\underline{u}(\bar{z})<u(\bar{z}) . \quad \|$

Proof of Proposition 1. As shown in Theorem 1, the equilibrium threshold function $z$ is such that

$$
\lim _{t=0^{+}} \phi(t, z(t))=0 \text { and } \lim _{t \rightarrow+\infty} \phi(t, z(t))=0,
$$

where

$$
\begin{aligned}
\phi(t, z(t))= & c-\rho \int_{0}^{\infty} \alpha[v(z(t)+w)-v(z(t))] G(d w)-\rho \int_{0}^{z(t)} \int_{z(t)-y}^{\infty} \underline{u}(z(t), y+w) G(d w) \frac{F(t, d y)}{F(t, z(t))} \\
& +\left(r+\rho \int_{0}^{z(t)}[1-G(z(t)-y)] \frac{F(t, d y)}{F(t, z(t))}\right)\left[\alpha v(z(t))-c_{0}\right] .
\end{aligned}
$$

Hence $z(t)$ decreases in $c$ and increases in $c_{0}$, in the limits for $t$ small and $t$ large, because

$$
\frac{\partial}{\partial c} \phi(t, z(t))=1>0 \text { and } \frac{\partial}{\partial c_{0}} \phi(t, z(t))=-\left(r+\rho \int_{0}^{z(t)}[1-G(z(t)-y)] \frac{F(t, d y)}{F(t, z(t))}\right)<0 .
$$

Consider now the ordinary differential equation characterizing the equilibrium threshold $z$ on the whole domain

$$
z^{\prime}(t)=\frac{\phi(t, z(t))}{\left[\alpha v(z(t))-c_{0}-\underline{u}(z(t), z(t))\right] \frac{f(t, z(t))}{F(t, z(t))}} .
$$

Making the dependence of $z(t)$ in cost $c$ explicit, suppose by contradiction that $z(t, c+\Delta c)>z(t, c)$ for some $t$ and some small $\Delta c$. As $z(t)$ decreases in $c$ for $t$ small and $t$ large, there must be at least two crossing points $t_{1}$ and $t_{2}$, such that $z\left(t_{1}, c+\Delta c\right)=z\left(t_{1}, c\right), z\left(t_{2}, c+\Delta c\right)=z\left(t_{2}, c\right)$ and $z_{1}\left(t_{1}, c+\Delta c\right) \geq z_{1}\left(t_{1}, c\right), z_{1}\left(t_{2}, c+\Delta c\right) \leq z_{1}\left(t_{2}, c\right)$, but for $\Delta c$ small,

$$
\left.\frac{d z_{1}(t, c)}{d c}\right|_{z(t, c)=z(t, c+\Delta c) \equiv z(t)} \approx \frac{\partial}{\partial c}\left[\frac{\phi(t, z(t))}{\left[\alpha v(z(t))-c_{0}-\underline{u}(z(t), z(t))\right] \frac{f(t, z(t))}{F(t, z(t))}}\right] \propto \frac{\partial \phi(t, z(t))}{\partial c}=1>0 .
$$

Analogously, the supposition that $z\left(t, c_{0}+\Delta c_{0}\right)>z(t, c)$ for some $t$ is contradicted by

$$
\begin{aligned}
&\left.\frac{d z_{1}\left(t, c_{0}\right)}{d c_{0}}\right|_{z\left(t, c_{0}\right)=z\left(t, c_{0}+\Delta c_{0}\right) \equiv z(t)} \approx \frac{\partial}{\partial c_{0}}\left[\frac{\phi(t, z(t))}{\left[\alpha v(z(t))-c_{0}-\underline{u}(z(t), z(t))\right] \frac{f(t, z(t))}{F(t, z(t))}}\right] \\
& \propto-\left(r+\rho \int_{0}^{z(t)}[1-G(z(t)-y)] \frac{F(t, d y)}{F(t, z(t))}\right)\left[\alpha v(z(t))-c_{0}-\underline{u}(z(t), z(t))\right]+\phi(t, z(t)) \\
&<-\left(r+\rho \int_{0}^{z(t)}[1-G(z(t)-y)] \frac{F(t, d y)}{F(t, z(t))}\right)\left[\alpha v(z(t))-c_{0}-\underline{u}(z(t), z(t))\right]<0 .
\end{aligned}
$$


Further, consider

$$
\frac{\partial}{\partial \alpha} \phi(t, z(t))=\left(r+\rho \int_{0}^{z(t)}[1-G(z(t)-y)] \frac{F(t, d y)}{F(t, z(t))}\right) v(z(t))-\rho \int_{0}^{\infty}[v(z(t)+w)-v(z(t))] G(d w),
$$

Suppose that $\underline{u}$ is small. Since $\phi(t, z(t)) \approx 0$, we obtain that

$$
\begin{aligned}
\frac{\partial}{\partial \alpha} \phi(t, z(t)) \approx & \left(r+\rho \int_{0}^{z(t)}[1-G(z(t)-y)] \frac{F(t, d y)}{F(t, z(t))}\right) v(z(t)) \\
& -\frac{1}{\alpha}\left[c+\left(r+\rho \int_{0}^{z(t)}[1-G(z(t)-y)] \frac{F(t, d y)}{F(t, z(t))}\right)\left[\alpha v(z(t))-c_{0}\right]\right] \\
\propto & \left(r+\rho \int_{0}^{z(t)}[1-G(z(t)-y)] \frac{F(t, d y)}{F(t, z(t))}\right) c_{0}-c
\end{aligned}
$$

This quantity is negative (positive) when $c$ is sufficiently large (small) relative to $c_{0}$.

Finally, we need to calculate, for $\underline{u}$ small,

$$
\begin{aligned}
& \left.\frac{d z_{1}(t, \alpha)}{d \alpha}\right|_{z(t, \alpha)=z(t, \alpha+\Delta \alpha) \equiv z(t)} \approx \frac{\partial}{\partial \alpha}\left[\frac{\phi(t, z(t))}{\left[\alpha v(z(t))-c_{0}-\underline{u}(z(t), z(t))\right] \frac{f(t, z(t))}{F(t, z(t))}}\right] \\
& \quad \approx \frac{\partial}{\partial \alpha}\left[\frac{c-\alpha \rho \int_{0}^{\infty}[v(z(t)+w)-v(z(t))] G(d w)+\left(r+\rho \int_{0}^{z(t)}[1-G(z(t)-y)] \frac{F(t, d y)}{F(t, z(t))}\right)\left[\alpha v(z(t))-c_{0}\right]}{\left[\alpha v(z(t))-c_{0}\right] \frac{f(t, z(t))}{F(t, z(t))}}\right] \\
& \propto \frac{\partial}{\partial \alpha}\left[\frac{c-\alpha \rho \int_{0}^{\infty}[v(z(t)+w)-v(z(t))] G(d w)}{\alpha v(z(t))-c_{0}}\right] \\
& \quad \propto-\rho \int_{0}^{\infty}[v(z(t)+w)-v(z(t))] G(d w)\left[\alpha v(z(t))-c_{0}\right] \\
& -v(z(t))\left[c-\alpha \rho \int_{0}^{\infty}[v(z(t)+w)-v(z(t))] G(d w)\right] \\
& \quad=c_{0} \rho \int_{0}^{\infty}[v(z(t)+w)-v(z(t))] G(d w)-c v(z(t)),
\end{aligned}
$$

where the first proportionality sign is found by dividing through by $\alpha v(z(t))-c_{0}$, and the second one by simplifying. For any $t, 0<\underline{z}<z(t)<\bar{z}<\infty, v^{\prime}>0, \underline{z}$ is bounded away from 0 and $\bar{z}$ is bounded away from $\infty$ for $c_{0}=0$ and $c=0$. Hence, the above quantity cannot change sign when $c$ is either sufficiently small or sufficiently large relative to $c_{0}$. $\quad \|$

Acknowledgment. We are very grateful for the effort of two referees and of the editor, Bernard Salanie', in making this paper publishable. The paper also benefitted from comments by Dino Gerardi, Philippe Jehiel, David Levine, Stephen Morris, Glenn Mac Donald, and Nicola Pavoni, and by audiences at the Decentralization Conference 2005, Society of Economic Dynamics, World Congress of the Econometric Society 2005, and at seminars at Harvard University, Yale University, Cambridge University, Edinburgh University and Leicester University. Further, Stefano DeMichelis' help in checking mathematical proofs and David Deller's help in checking the verbal presentation are gratefully acknowledged. The usual caveat applies. Hugo Hopenhayn for NSF support under grants SES-0215764 and SES-0922461.

\section{REFERENCES}

ABREU, D. and BRUNNERMEIER, M. (2003), "Bubbles and Crashes", Econometrica, 71, 173-204.

BAYE, M., KOVENOCK, D. and DE VRIES, C. G. (1993), "Rigging the Lobbying Process: An Application of the All-Pay Auction", American Economic Review, 83, 289-294.

BERGEMANN, D. and VALIMAKI, J. (1996), "Learning and Strategic Pricing", Econometrica, 64, 1125-1150.

BERGEMANN, D. and VALIMAKI, J. (2000), "Experimentation in Markets", Review of Economic Studies, 67, 213-234. 
BINMORE, K. (2004), Fun and Games: A Text on Game Theory, 2nd edn (Florence, KY: Houghton Mifflin College Div).

BOLTON, P. and HARRIS, C. (1999), "Strategic Experimentation", Econometrica, 67, 349-374.

BULOW, J. and KLEMPERER, P. (1999), "The Generalized War of Attrition", American Economic Review, 89, $175-189$.

DÉCAMPS, J. P. and MARIOTTI, T. (2004), "Investment Timing and Learning Externalities", Journal of Economic Theory, 118, 80-102.

DIXIT, A. K. and PINDYCK, R. S. (1994), Investment Under Uncertainty (Princeton, NJ: Princeton University Press).

FUDENBERG, D., GILBERT, R., STIGLITZ, J. and TIROLE, J. (1983), "Preemption, Leapfrogging and Competition in Patent Races", European Economic Review, 22, 3-31.

FUDENBERG, D. and TIROLE, J. (1985), "Preemption and Rent Equalization in the Adoption of New Technology", Review of Economic Studies, 52, 383-401.

FUDENBERG, D. and TIROLE, J. (1986), "A Theory of Exit in Duopoly”, Econometrica, 54, 943-960.

HALL, B. H., JAFFE, A. B. and TRAJTENBERG, M. (2001), "The NBER Patent Citation Data File: Lessons, Insights and Methodological Tools" (Working Paper No. 8498, NBER).

HALL, B. H. and ZIEDONIS, R. H. (2001), "The Determinants of Patenting in the U.S. Semiconductor Industry, 1980-1994", Rand Journal of Economics, 32, 101-128.

HARRIS, C. and VICKERS, J. (1985), "Perfect Equilibrium in a Model of a Race", Review of Economic Studies, 52, 193-209.

HARRIS, C. and VICKERS, J. (1987), "Racing with Uncertainty", Review of Economic Studies, 54, 1-21.

KARLIN, S. (1959), The Theory of Infinite Games, Vol. 2 (New York: Dover Publications).

KELLER, G., CRIPPS, M. and RADY, S. (2005), "Strategic Experimentation with Exponential Bandits", Econometrica, 73, 39-68.

KELLER, G. and RADY, S. (1999), “Optimal Experimentation in a Changing Environment”, Review of Economic Studies, 66, 475-507.

KELLER, G. and RADY, S. (2003), "Price Dispersion and Learning in a Dynamic Differentiated Goods-Duopoly", RAND Journal of Economics, 34, 138-165.

KORTUM, S. and LERNER, J. (1999), "What is Behind the Recent Surge in Patenting?", Research Policy, 28, 1-22.

KRISHNA, V. and MORGAN, J. (1997), "An Analysis of the War of Attrition and the All-Pay Auction", Journal of Economic Theory, 72, 343-362.

LAMBRECHT, B. M. and PERRAUDIN, W. R. (2003), "Real Options and Preemption under Incomplete Information", Journal of Economic Dynamics and Control, 27, 619-643.

LIPPMAN, S. A. and MCCARDLE, K. F. (1988), "Preemption in R\&D Races", European Economic Review, 32, 1661-1669.

MOSCARINI, G. and SQUINTANI, F. (2004), "Competitive Experimentation with Private Information" (Mimeo, Yale University).

MURTO, P. and VALIMAKI, J. (2005), "Experimentation and Observational Learning in a Market with Exit" (Mimeo, University of Helsinki).

REINGANUM, J. (1981), “Dynamic Games of Innovation”, Journal of Economic Theory, 25, 21-41.

RIORDAN, M. (1992), "Regulation and Preemptive Technology Adoption”, RAND Journal of Economics, 23, 334-349.

ROSENBERG, D., SOLAN, E. and VIEILLE, N. (2007), "Social Learning in One-Arm Bandit Problems", Econometrica, 75, 1591-1611.

ROSENTHAL, R. (1982), "Games of Perfect Information, Predatory Pricing and the Chain Store Paradox", Journal of Economic Theory, 25, 92-100.

ROSS, S. (1995), Stochastic Processes (New York: Wiley).

SIMON, L. and STINCHCOMBE, M. (1989), "Extensive Form Games in Continuous Time, Part I: Pure Strategies", Econometrica, 57, 1171-1214.

WEEDS, H. (2002), "Strategic Delay in a Real Options Model of R\&D Competition", Review of Economic Studies, 69 , 729-747. 